\title{
Brain mechanisms for changes in processing of conditioned stimuli in Pavlovian conditioning: Implications for behavior theory
}

\author{
PETER C. HOLLAND \\ Duke University, Durham, North Carolina
}

\begin{abstract}
This article is a review of the results of a series of experiments designed to identify brain systems involved in appetitive conditioning of rats. It discusses some of their implications for behavioral theories of learning, especially those that concern changes in processing of conditioned stimuli (CSs). Evidence is presented which suggests that separable brain circuits are involved in (1) the production of CS-dependent conditioned orienting responses, (2) the enhancement of CS associability produced when expectancies about upcoming events are violated, (3) the reduction of CS associability produced when stimuli are consistent predictors of other events or are presented without consequence, and (4) the abilities of CSs to serve as reinforcers for second-order conditioning and to be sensitive to postconditioning changes in the value of the unconditioned stimulus (US). Finally, none of these circuits seems critical for normal acquisition of the most common indicator of Pavlovian conditioning, US-dependent conditioned responses (CRs). Although the independence of brain pathways does not demand independence of behavioral function, clustering of behavioral phenomena on anatomical grounds may provide useful guides for constructing behavior theories.
\end{abstract}

Pavlovian conditioning alters the way in which animals process events. In a typical Pavlovian conditioning experiment, a relatively neutral conditioned stimulus (CS) is repeatedly paired with a motivationally significant unconditioned stimulus (US). The most frequently described result of these pairings is the emergence of the CS's ability to elicit a conditioned response (CR) that resembles the unconditioned response (UR) to the US. But it has long been recognized that the arrangement of Pavlovian relations among events may produce a variety of consequences.

These diverse products of learning can be categorized in many ways. One popular distinction is to contrast learning sequellae that reflect the strength of an association between internal representations of the CS and the US with more general effects on CS processing, which may be only loosely related to a CS's associative strength. Examples of the former category include the CS's ability to elicit CRs whose form depends on the US (see, e.g., Rescorla \&

The research described in this article was supported by grants from the National Institute of Mental Health and the International Human Frontier Science Program to Duke University and the University of North Carolina. Thanks are due Michela Gallagher, with whom these ideas and research were developed. I also thank Mark Baxter, David Bucci, Lindsey Ciali, Michael Conley, Derick Davis, Greg Fox, JungSoo Han, Javier Morell, and Jennifer Thornton for permission to describe their unpublished data. Requests for reprints should be sent to P. C. Holland, Department of Psychology: Experimental, Duke University Box 90086, Durham, NC 27708-0086 (e-mail: pch@duke.edu).

Note: This article is one of those occasionally invited by the editor in which authors have the opportunity to provide an overview of their research programs. - Editor
Solomon,1967), its capacity to serve as a reinforcer for new learning in Pavlovian second-order conditioning or operant secondary reinforcement procedures (Rescorla, 1980), and its power to control motivational processes that may broadly modulate other learned (e.g., Mowrer, 1960; Rescorla \& Solomon, 1967) or unlearned (e.g., Weingarten, 1983) behavior. Examples of the latter category include changes in the associability or salience of the CS (e.g., Mackintosh, 1975; Pearce \& Hall, 1980), changes in perceptual processing of the CS (e.g., Edeline \& Weinberger, 1992), and changes in a CS's abilities to control orienting behavior (e.g., Kaye \& Pearce, 1984).

In this review, I describe a series of experiments conducted in collaboration with Michela Gallagher and several colleagues in our laboratories. The results of these experiments show that different brain pathways are involved in the display of many of these products of learning. Although the independence of brain pathways does not in itself demand independence of behavioral function, these findings may be useful for further development of behavioral theory. Most of the discussion in this article concerns the implications of our data for theories of attentional function in Pavlovian conditioning. Little is said about the brain systems themselves; the interested reader is referred to a brief review by Gallagher and Holland (1994).

The first section of this review briefly introduces the logic of the methods that we use to study brain-behavior relations. In the second section, I discuss evidence that the brain systems involved in the generation of CS-dependent conditioned orienting responses (ORs) are separable from those responsible for the generation of US-dependent CRs. Third, I outline studies of the brain circuitry that mediates 
a CS's ability to participate in various functions often loosely described as motivational. These functions include a CS's ability to serve as a reinforcer for secondorder conditioning, its ability to modulate consummatory behavior, and its sensitivity to postconditioning changes in the value of the US. The results of these studies suggest that the brain mechanisms involved in a CS's acquisition of motivational value are distinct from those involved in its acquisition of the ability to elicit CRs. Fourth, I discuss experiments in which we have investigated brain mechanisms for changes in the associability of CSs that occur as a result of various conditioning treatments. These studies indicate that increases and decreases in CS associability depend on different brain systems. Finally, I discuss some implications of these data for behavior theory.

\section{Use of Lesion Techniques to Infer Brain-Behavior Relations}

In most of the studies discussed in this article, we used selective neurotoxic lesion techniques, in which the performance of rats with damage to relatively discrete cell groups in the brain is compared with that of intact subjects. Involvement of a given brain region in some behavioral function is inferred if lesioned subjects show deficits in performance on tasks thought to involve that function but show no evidence of deficiencies in performance on tasks thought not to involve that function. Frequently, these studies show double dissociations, in which performance on one task is affected by lesion A but not by lesion B, whereas performance on another task is affected by lesion $B$, but not by lesion A. Because the same behavioral response is usually measured in both lesion-affected and -unaffected tasks, lesion-induced deficits in performance are more readily attributed to specific functional deficits rather than to more general motor or motivational processes.

The use of lesion methods to infer brain-behavior relations is frequently criticized (see, e.g., Luria, 1966; Staddon, 1983). The general complaint is that damaging part of a complex system may disrupt that system's normal mode of operation without yielding much information about it. A common analogy is that of using a hammer to study the functions of radio or television components. Smashing one component may eliminate the sound, for example, and smashing another may generate squeals; but inferences that those components are, respectively, the seats of sound production and squeal-suppression functions are unwarranted. Alternately, in a radio with redundant functions (e.g., stereo sound), one might eliminate function of a circuit with little evidence of deficits in the radio's performance: destruction of a circuit responsible for stereo sound might leave the radio able to produce perfect monaural sound.

Although such criticisms cannot be logically ruled out, their impact is considerably reduced when both the neurobiological and behavioral methods are more sophisticated. For example, the more discrete the brain damage, the more likely that the disruption it produces will be precisely specifiable. Many lesion techniques used today are relatively selective, often damaging only particular classes of cells in particular locations, leaving intact passing nerve fibers and even other types of neurons intermingled with the target neurons. Similarly, the selection of lesion sites is guided by better knowledge of patterns of anatomical and functional connectivity among brain regions.

Equally critical to the interpretation of the results of lesion studies is the sophistication of the behavioral techniques used. One issue is that of the specificity of behavioral deficits observed after brain lesions. The occurrence of general behavioral deficits after a lesion is usually not particularly informative: the subject may have deficits in sensory or motor abilities, motivational function, learning ability, and so forth, that are difficult to specify from the experiment at hand. But deficits confined to particular behaviors under well-specified conditions can be quite informative. A second issue concerns the availability of multiple assessments of the same putative behavioral deficiency. For example, the claim that region A is importantly involved in function $\mathrm{X}$ is more convincing if performances on several independent tasks thought to involve function $\mathrm{X}$ are all disrupted by destruction of region $\mathrm{A}$. Multiple-item psychological tests inspire more confidence than single-item tests do. In most cases, the availability of multiple assessment techniques is intertwined with the existence of more sophisticated theories of behavioral function that can more precisely specify the conditions under which some process may be engaged. Thus, in some sense, lesion techniques can be more effective when applied within more coherent theories of psychological function.

Finally, it must be recognized that the use of lesion methods is not confined to the problem of understanding the function of the missing (lesioned) brain region. Indeed, it can be argued that lesion methods make a major contribution to the understanding of the operation of the remaining parts of the system (see, e.g., Teitelbaum \& Pellis, 1992). For example, in this article, I argue that brain systems involved in changes in CS associability are separable from those involved in reinforcement processes such as those specified by the Rescorla-Wagner (1972) model. Interestingly, we have found that performance of rats with lesions to the former brain systems is often more consistent with the predictions of the Rescorla-Wagner model (which does not incorporate changes in CS associability) than is the performance of intact rats. Conditioning procedures used to support so-called attentional or CS-processing theories (e.g., Pearce \& Hall, 1980) because they generate performances inconsistent with the predictions of the Rescorla-Wagner model in intact rats often yield RescorlaWagner-like outcomes in lesioned rats. Thus, rats with these lesions might be used to study the operation of reinforcement systems such as those specified by the RescorlaWagner model, without the confounding influences of changes in CS processing.

\section{Amygdala Systems in the Conditioning of Orienting Responses}

Holland (1977; see Holland, 1984a, for a review) found that Pavlovian CS-food pairings resulted in the display of 
two classes of conditioned behavior in hungry rats. The first was behavior appropriate to the US (e.g., approaching and contacting the food cup), and the second was an enhanced orienting response (OR) specific to the type of CS used (e.g., rearing on the hind legs and orientation toward visual CSs, and a startle response to auditory CSs). Although before CS-US pairings US-dependent behaviors (food cup) seldom occurred during the CSs, the ORs (rear and startle) occurred at moderate levels. Without CS-US pairings, these ORs habituated rapidly but were enhanced above their original levels with CS-US pairings.

Considerable behavioral evidence suggested that these two classes of behavior were often separable. For example, whereas the ORs occurred during the initial portions of the CS-US interval, the CRs were generally more frequent during later portions of that interval, nearer the time of US delivery (Holland, 1977, 1980a). Second, changes in the nature of the CS (e.g., alterations in modality, localizability, location, etc.) produced parallel changes in the form of behavior observed early in the CS interval but had little effect on the form of behavior later in the interval (Holland, 1977, 1980b). At the same time, the opposite patterns of data were observed when properties of the US (e.g., delivery location, nature of food cup, liquid or solid food) were varied (Holland, 1979b). Third, if CS onset (the reliable generator of an OR before conditioning) was made less relevant to the delivery of the US, by extending the CS-US interval, or by intermixing short, nonreinforced CSs with longer, reinforced ones, the frequency of conditioned ORs was substantially reduced (Holland, 1980a). Finally, several experimental manipulations, such as varying US magnitude or probability, the imposition of omission contingencies, and the postconditioning devaluation of the US differentially affected the frequencies of these two classes of conditioned behavior (Holland, 1979a, 1979b; Holland \& Straub, 1979).
Much of our work on the brain mechanisms of appetitive conditioning grew from an initial observation that, with simple conditioning procedures, brain regions critical to the emergence of conditioned ORs are apparently not involved in the acquisition of US-dependent CRs. These findings reinforced the results of the previous behavioral work in implying some degree of independence of these two classes of conditioned behavior. In the remainder of this section, I will describe the experiments that both led to this conclusion and set the stage for many of our further studies.

In our first experiment (Gallagher, Graham, \& Holland, 1990), we found that neurotoxic damage to the amygdala central nucleus $(\mathrm{CN})$ produced a profound deficit in the acquisition of Pavlovian conditioned ORs to both auditory and visual cues but preserved the acquisition of USdependent conditioned food cup behavior. Hungry rats in standard conditioning chambers initially received brief preexposure to auditory (tone) and visual (light) stimuli. Then the rats received conditioning sessions in which presentations of one stimulus were followed by delivery of food pellets to a cup recessed in one wall of the chamber, and presentations of the other cue were nonreinforced. Conditioning was assessed by observation of videotapes made of the rats' behavior, using a time-sampling technique (see Holland, 1977). Rear behavior was scored whenever a rat had both front feet off the floor of the experimental chamber but was not grooming. Food cup behavior was scored whenever any portion of a rat's head was visible within the recessed food cup. The behavioral measure "percentage total behavior" was computed for each behavior by dividing the number of observations on which that behavior was scored in some interval (e.g., during the $\mathrm{CS}$ ) by the total number of observations made in that interval. Because the observations occurred at a constant rate throughout the studies, this index resembles an absolute frequency measure.

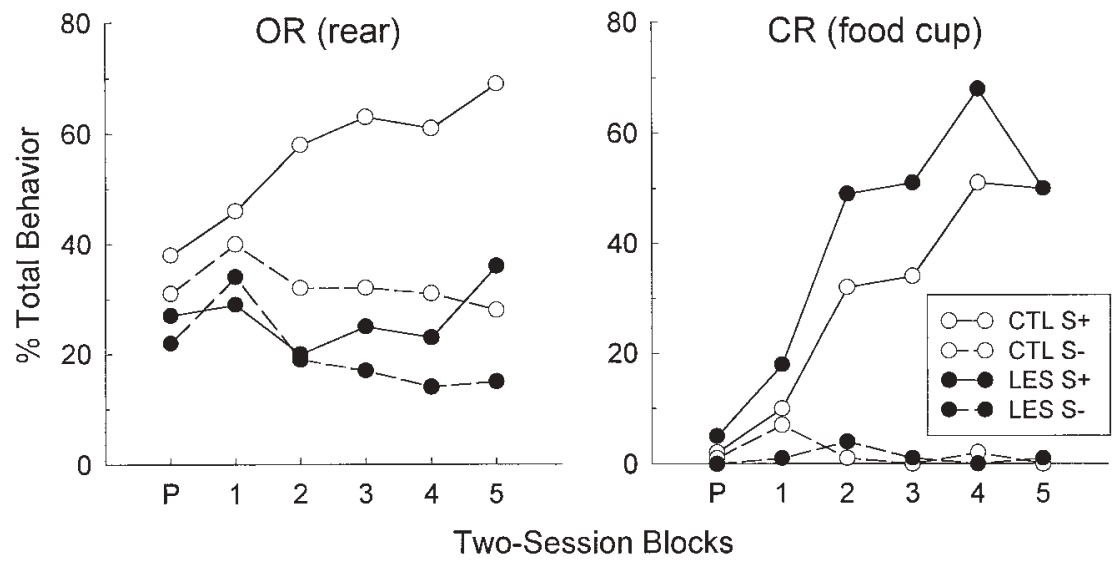

Figure 1. Acquisition of conditioned rear (left panel) and food cup behaviors (right panel) to reinforced $(\mathrm{S}+)$ and nonreinforced $(\mathrm{S}-)$ visual conditioned stimuli in the conditioning phase of Gallagher, Graham, and Holland's (1990) study. OR, conditioned orienting (rear) responses; CR, conditioned food cup responses; LES, rats with lesions of amygdala central nucleus $(\mathrm{CN})$; CTL, unlesioned control rats. $P$ on the abscissae refers to the final session of nonreinforced preexposure to the visual cue, given prior to the conditioning phase. 
We found that bilateral lesions of the $\mathrm{CN}$ produced by microinjections of the neurotoxin ibotenic acid reduced conditioning of CS-specific orienting behaviors but left acquisition of food cup related behaviors unimpaired. Figure 1 shows the results of this experiment for the visual cue only; the results with the auditory cues were comparable. In this and all subsequent portrayals of data with visual CSs, the frequency of ORs is shown for the first $5 \mathrm{sec}$ of the 10-sec cues and the frequency of food cup behavior is shown for the second 5-sec interval. Because nearly all conditioned ORs occur within the first 5-sec interval, this method of reporting the data yields both the most appropriate assessment of conditioned ORs and a measure of CRs that is relatively uncontaminated by competition between CRs and ORs (see Holland, 1977, and Gallagher et al., 1990).

The lack of any effect of the lesion on food cup CRs made it unlikely that the deficit in conditioned orienting behavior reflected some general learning or motivational deficiency (a point considered in more detail in the next section of this article). Furthermore, although the topography of the conditioned OR was similar to that of the unconditioned OR to the CS when it was initially presented, $\mathrm{CN}$ damage did not alter the form or frequency of unconditioned ORs in the preexposure phase or the habituation of those responses with repeated nonreinforced presentation in that phase. Thus, the observed deficit in the acquisition of conditioned ORs in CN-lesioned rats did not reflect an inability to orient to the cues, or some general motor deficit. Instead, it appeared that the $\mathrm{CN}$ was involved very selectively in the development of learned orienting responses.

We considered two alternative implications of our finding that the amygdala $\mathrm{CN}$ is importantly involved in the production of conditioned ORs, both of which are consistent with what is known about amygdala function and the amygdala's connections with other brain systems. One possibility is that the learned potentiation of ORs involves the CN's modulation of the activity of lower level circuitry involved in the production of particular ORs. Many brainstem targets of the $\mathrm{CN}$ exert control over autonomic and behavioral ORs that occur in conditioning experiments. For example, changes in heart rate (a frequent component of orienting) and conditioning-dependent potentiation of the startle reflex appear to depend on projections from the $\mathrm{CN}$ to those brainstem systems (see, e.g., Hitchcock \& Davis, 1991; Kapp, Pascoe, \& Bixler, 1984; LeDoux, Iwata, Cicchetti, \& Reis, 1988). Alternately, the CN's involvement in conditioned orienting might reflect considerably more widespread changes in the processing of CSs, which are mediated by higher level brain systems. A variety of these systems, often assigned roles in arousal, vigilance, and attention, receive input from the amygdala CN (see, e.g., Grove, 1988; Heimer \& Alheid, 1991; Price \& Amaral, 1981).

Considerable evidence from our laboratories supports the latter possibility. First, many of these studies (discussed in section 4 of this article) show that the $\mathrm{CN}$ indeed has effects on CS processing that go beyond modulation of conditioned ORs and that these effects are mediated by the CN's connections with forebrain systems. Second, Han, McMahan, Holland, and Gallagher (1997) recently showed that the emergence of conditioned ORs also depends on the CN's connections with forebrain regionsspecifically, the dorsolateral striatum. $\mathrm{CN}$ neurons project to dopaminergic neurons in the substantia nigra pars compacta, which in turn project mostly to the dorsolateral striatum. It has often been claimed that the dorsolateral striatum plays an important role in the regulation of orienting behavior to stimuli of many modalities (see, e.g., Carli, Jones, \& Robbins, 1989).

Han et al. (1997) found that functional disconnection of this $\mathrm{CN}$-striatal pathway produced changes identical to those that Gallagher et al. (1990) found with CN lesions. They exploited the apparently exclusively ipsilateral connections between these two regions to show that the connection between the $\mathrm{CN}$ and striatum, rather than the separate contribution of each of those structures, is critical to the acquisition of conditioned ORs. The performance of rats with unilateral damage to either structure alone was unimpaired, but rats with damage to one structure on the right side and the other structure on the left side (thus preventing CN-striatal information flow on both sides, but leaving adequate function of each structure separately) showed no evidence for the acquisition of conditioned ORs. At the same time, these dual-lesioned rats were unimpaired in their display and habituation of unconditioned ORs and in their acquisition of UR-like food cup CRs. Thus, production of learned ORs apparently depends on the coordinated action of both the $\mathrm{CN}$ and the dorsolateral striatum (via the substantia nigra).

Interestingly, the CN's control over the display of conditioned ORs and its control over other, more global, changes in CS processing seem to be exerted via separate brain pathways. In the research described in the remaining sections, we examined brain circuitry involved in other aspects of CS processing, including incentive motivation (section 3) and both incremental and decremental changes in the associability of CSs (section 4). These functions seem to be independent of each other and of the acquisition of UR-like CRs in conditioning.

\section{Amygdala Systems and Incentive Motivation}

Many researchers have implicated the amygdala in the assignment of emotional significance or value to events through associative learning (see Gallagher \& Chiba, 1996, for a brief recent review). For example, in animals with amygdala damage, a cue that signals an impending aversive event (such as an electric shock) fails to elicit behavior characterized as "fearful" (see, e.g., Davis, 1992; LeDoux, 1992). Similarly, much evidence suggests that cues paired with appetitive events are less likely to acquire positive value in rats with amygdala damage. For example, intact rats learn to prefer environmental locations consistently paired with food, but rats with damage to the lateral 


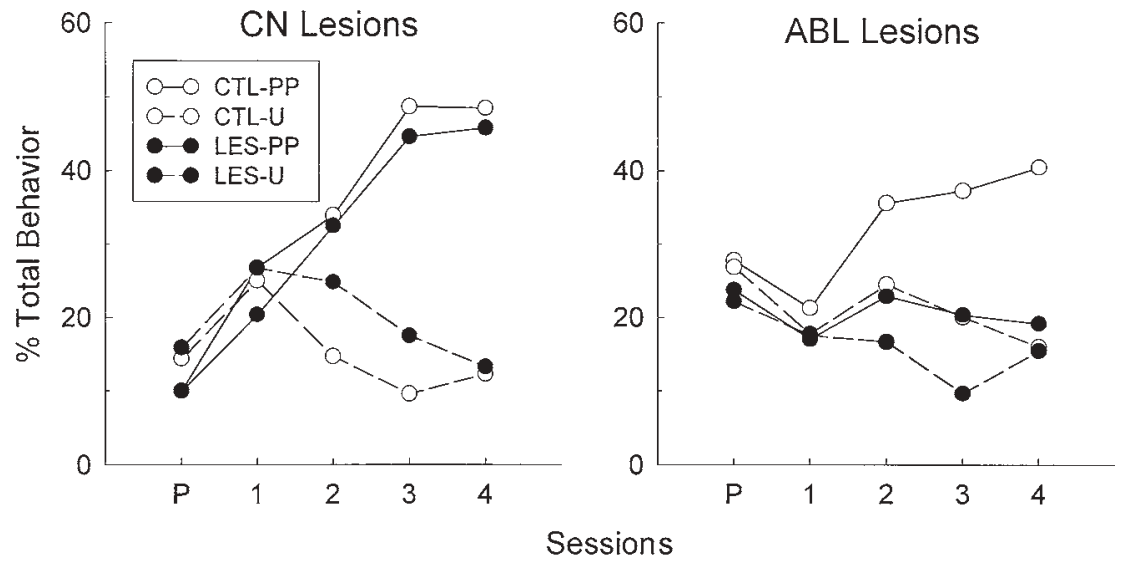

Figure 2. Acquisition of second-order conditioned food cup responding to a tone conditioned stimulus in experiments reported by Hatfield, Han, Conley, Gallagher, and Holland (1996, Experiments 1 and 3). The left panel shows responding of rats that received lesions (LES) of the amygdala central nucleus $(\mathrm{CN})$, and the right panel shows responding of rats that received lesions of the basolateral amygdala (ABL). In each experiment, unlesioned control (CTL) rats received microinjections of a nontoxic vehicle into the same brain region that was destroyed in the lesioned rats. PP refers to rats that received both light-food and tone-light pairings, and $U$ refers to rats that received either light-food or tone-food pairings, but not both. $P$ on the abscissae refers to responding during a pretest of responding to the tone alone.

(McDonald \& White, 1993) or basolateral (Cador, Robbins, \& Everitt, 1989) nuclei of the amygdala fail to show such learned place preferences.

In contrast, the results of our experiments suggest that the amygdala $\mathrm{CN}$ is not important for this acquisition of motivational value or significance by CSs. In the absence of manipulations designed to alter attentional processing (see section 4), lesion-induced deficits were confined to conditioned orienting behavior; conditioned approach to the food cup during the CS was unaffected (e.g., Gallagher et al., 1990). Furthermore, the magnitude of these CRs tracked changes in the magnitude of the USs (Holland \& Gallagher, 1993b, described in section 4). To examine more directly the role of the amygdala $\mathrm{CN}$ in the transfer of motivational significance from US to CS, we did three additional experiments with $\mathrm{CN}$-lesioned rats. Each experiment was designed to reveal a different aspect of the coding of motivational value by CSs. In the first experiment, we assessed a CS's ability to modulate food consumption; in the second, a CS's ability to serve as a reinforcer for secondorder conditioning; and in the third, a CS's sensitivity to postconditioning devaluation of the food US.

At the same time, in two of these tasks, we also examined the effects of lesions of another region of the amygdala, the basolateral amygdala (ABL). Other investigators have found that lesions of this brain region left Pavlovian CSs unable to serve as conditioned reinforcers in the secondary reinforcement of a new operant response (e.g., Cador et al., 1989). Similarly, as noted earlier, animals with nonspecific damage to the whole amygdala often show motivational dysfunctions. The results described in this section reconcile the two literatures by showing that although damage confined to the amygdala $\mathrm{CN}$ had no effects on presumably motivational aspects of CS function, damage to the ABL produced substantial deficits.

$\mathrm{CN}$ lesions and CS-potentiated eating. Food-sated rats can be induced to eat by presenting a previously trained signal for food (Weingarten, 1983). One interpretation of this finding is that the signal, because of its association with the US, engages a conditioned incentive motivational process that energizes feeding behavior. Gallagher and Holland (1992) considered whether rats with $\mathrm{CN}$ lesions would show a similar effect. After repeated pairings of a CS with food pellets while rats were food deprived, the rats were allowed to return to their original adlib weights for several days and were given free access to the food pellets previously used as the US. Their readiness to eat the pellets was then tested in two sessions in which 20 food pellets were made available. In one session, the CS was presented periodically; in the other, no CSs were presented. Both lesioned and intact rats ate considerably more during the session in which CSs were presented. In the CS session, lesioned rats ate an average of 16.9 pellets and intact rats consumed 15.2, whereas in the no-CS session, lesioned rats consumed 3.8 pellets and intact rats ate 0.2 . Thus, $\mathrm{CN}$ damage had no effect on the ability of an appetitive $\mathrm{CS}$ to enhance food consumption.

Amygdala lesions and second-order conditioning. Perhaps the most frequently cited measure of a CS's changed motivational value as a consequence of its pairing with food is its acquired ability to reinforce subsequent learning. In our first experiment in this series (Hatfield, Han, Conley, Gallagher, \& Holland, 1996, Experiment 3), we examined a CS's ability to support second-order con- 

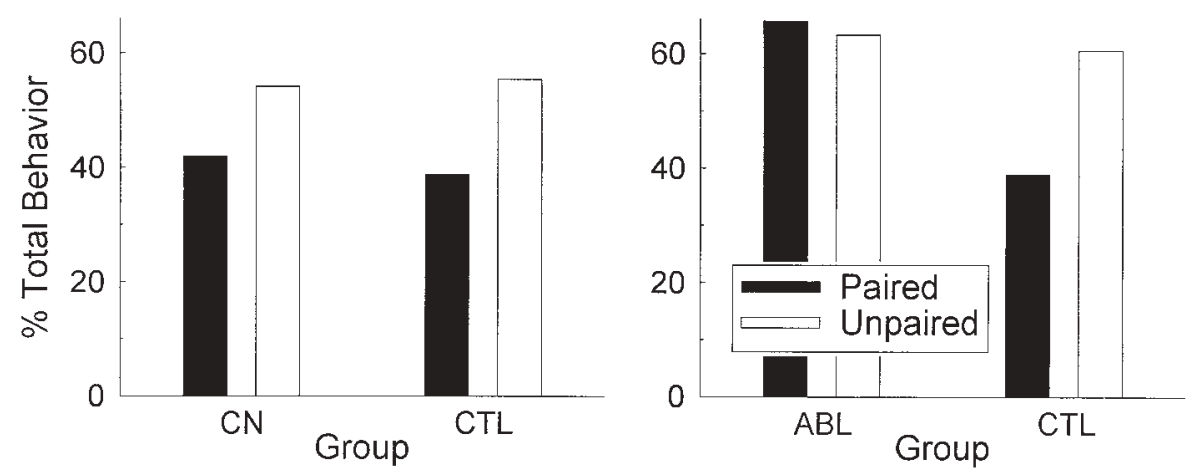

Figure 3. Performance of food cup conditioned responses during a light conditioned stimulus after food devaluation treatment in experiments reported by Hatfield, Han, Conley, Gallagher, and Holland (1996, Experiments 2 and 4). In these experiments, all rats first received light-food pairings. Paired rats then received food-toxin pairings and unpaired rats received food and toxin $8 \mathrm{~h}$ apart. Finally, responding during the light alone was tested. The left panel shows responding of rats that received lesions of the amygdala central nucleus $(\mathrm{CN})$, and the right panel shows responding of rats that received lesions of the basolateral amygdala (ABL). In each experiment, unlesioned control (CTL) rats received microinjections of a nontoxic vehicle into the same brain region that was destroyed in the lesioned rats.

ditioning in rats with $\mathrm{CN}$ damage. Intact and $\mathrm{CN}$-lesioned rats were first given pairings of a light with food, which presumably allowed the light to acquire reinforcing value. Then, experimental rats received tone $\rightarrow$ light pairings, in the absence of food, whereas control rats received explicitly unpaired presentations of the tone and light. Additional control rats received tone-light pairings in the second phase, but received unpaired presentations of the light and food in the first phase. Both lesioned and intact rats in the experimental condition acquired robust secondorder conditioning to the tone CS (Figure 2, left panel). At the same time, the levels of conditioned ORs elicited by both the first- and second-order CSs were reliably lower in the $\mathrm{CN}$-lesioned rats than in the unlesioned rats (not shown). Thus, the CN pathways important for the display of conditioned ORs are apparently not importantly involved in the transfer of reinforcing value from the food US to the light CS in conditioning.

In contrast, in another experiment, with identical procedures (Hatfield et al., 1996, Experiment 1), rats with neurotoxic damage to the basolateral amygdala (ABL) failed to acquire second-order conditioning (Figure 2, right panel). At the same time, the ABL-lesioned and unlesioned rats showed the same levels of food cup CR and conditioned ORs (rearing) during the visual first-order CS. Thus, the ABL lesion deficit was specific to the acquisition of second-order conditioning. Although it could be argued that this outcome implies that a mechanism for the formation of second-order, but not first-order, associations involves the basolateral amygdala, we favored the possibility that the CS-US pairings did not enable the first-order CSs to gain access to the value of the US. Consequently, those CSs were ineffective as conditioned reinforcers. This interpretation was considered further in studies of US devaluation, described later in this section.
Similar conclusions about the role of the $\mathrm{CN}$ and $\mathrm{ABL}$ in acquired reinforcement and $\mathrm{CR}$ elicitation functions can be derived from a set of operant secondary reinforcement studies conducted in the laboratories of Trevor Robbins and Barry Everitt. In those experiments (Cador, et al., 1989; Robledo, Robbins, \& Everitt, 1996), rats first received Pavlovian CS-food pairings. Then, the delivery of that CS was made contingent on the performance of a particular operant response. Rats with ABL lesions failed to acquire the secondarily reinforced operant response, whereas rats with $\mathrm{CN}$ lesions and intact rats acquired that operant at similar rates. At the same time, Pavlovian food cup responses to the Pavlovian CS that served as the secondary reinforcer were unaffected by either lesion.

Amygdala lesions and US devaluation. In a final pair of experiments, US devaluation techniques were used in order to provide more support for the claim that although ABL damage interferes with the CS's acquisition of reinforcement value, $\mathrm{CN}$ damage does not. Holland and Straub (1979) and Holland (1990) showed that responding in the conditioning preparation used here is mediated by the value of some representation of the US at the time of performance. That is, after CS-US conditioning was completed, spontaneous changes in the CR elicited by the CS were observed if the value of the US was changed. For example, in one experiment (Holland, 1990), after pairings of two different tones with food USs of two different flavors, one food was devalued by pairing it with a toxin, $\mathrm{LiCl}$, in the absence of the CSs. Subsequent testing of responding to the CSs alone showed less responding to the CS whose formerly associated US had been devalued with $\mathrm{LiCl}$.

In these experiments, we asked whether rats with $\mathrm{CN}$ or ABL lesions also showed sensitivity to the current value of the US. In the first of these studies (Hatfield et al., 1996, Experiment 4), both CN-lesioned and intact rats 
first received light $\rightarrow$ food pairings. Then, some rats received two home cage pairings of the food (in dishes) with injection of $\mathrm{LiCl}$, whereas other rats received the food and $\mathrm{LiCl} 8 \mathrm{~h}$ apart. Both lesioned and unlesioned rats that received food-LiCl pairings showed large and equivalent reductions in food consumption, compared with the rats that received food and $\mathrm{LiCl}$ unpaired. More important, when the rats were returned to the conditioning chambers and presented with light CSs without food, lesioned and unlesioned rats that had received food-LiCl pairings showed similar reductions in conditioned food cup behavior, compared with the unpaired rats (Figure 3, left panel). Thus, CN lesions apparently did not alter the CS's ability to gain access to the current value of the US in conditioning.

However, in an experiment with the same procedures (Hatfield et al., 1996, Experiment 2), rats with ABL lesions failed to show spontaneous changes in their CRs to the light when the US was devalued (Figure 3, right panel). Nevertheless, both lesioned and unlesioned rats acquired the aversion to the food US at the same rate, and both showed the same level of aversion to that food in a final test of food consumption measured in the experimental chambers. Thus, although both light-food and food-LiCl conditioning were unimpaired by the ABL lesions, rats with ABL lesions seemed unable to alter their CRs to reflect the current value of the US. One interpretation of this observation, which is consistent with the failure of ABLlesioned rats to show second-order conditioning, is that in ABL-lesioned rats, the CS does not have access to the motivational significance of the US.

Taken together, the results of the experiments described in this section encourage several important conclusions. First, the $\mathrm{CN}$ is not an essential part of neural pathways involved in the transfer of value or motivational significance in conditioning. $\mathrm{CN}$ lesions did not affect a CS's ability to potentiate food consumption, to serve as a reinforcer for second-order conditioning, or to track postconditioning changes in the value of the US. These results are significant for our other investigations of $\mathrm{CN}$ function because they suggest that the behavioral deficits in responding to CSs displayed by $\mathrm{CN}$-lesioned rats are not likely to be the consequence of changes in the motivational significance of those CSs. These deficits include both the lack of conditioned ORs (section 2) and other response deficits that we will attribute (in section 4) to impairments in attentional processing. Second, the ABL is important for the display of some of these motivational functions of CSs. Lesions of this region prevented CSs from either serving as reinforcers for second-order conditioning or tracking postconditioning changes in US value. Third, the $\mathrm{CN}$, but not the ABL, is critical to a CS's ability to display conditioned ORs. Finally, neither the $\mathrm{CN}$ nor the ABL is importantly involved in the production of food cup CRs in first-order conditioning. Thus, at least three aspects of CS function - the ability to elicit US-dependent CRs, the power to elicit conditioned ORs, and the capacity to control motivational function - are apparently quite indepen- dent. Although in normal rats all three covary, in CN-lesioned rats the OR-evoking powers of a CS did not covary with its CR-evoking and motivational powers, and in ABL-lesioned rats, the motivational powers of a CS did not covary with its $\mathrm{CR}$ - and OR-eliciting powers.

\section{Brain Systems for Changes in the Associability of Conditioned Stimuli}

Many modern learning theories claim that conditioning experiences alter attention to CSs. Within these theories, CS-US pairings not only establish CS-US associations, but also change subjects' attentional processing of the CS. Theories that give an important role to changes in processing of the CS differ in their views of both the nature of these attentional changes and the environmental conditions or contingencies that induce them.

Consider, first, the nature of attentional changes. Perhaps the most elementary distinction is one between incremental and decremental changes in processing. For example, Sutherland and Mackintosh (1971) and Mackintosh (1975) proposed that animals learn to attend to relatively good predictors of reinforcement but learn to ignore relatively poor predictors of reinforcement. Although learning to attend and learning to ignore have typically been construed as symmetrical processes (see, e.g., Mackintosh, 1975), later in this section we will present evidence that increases and decreases in CS processing are subserved by different neural systems.

An equally basic, but sometimes more subtle, distinction in the nature of changes in attention concerns the processing of CSs in the acquisition of new learning and in the performance of previously acquired CRs. What are the consequences of increases or decreases in attention to a CS? Not surprisingly, most learning theories concentrate on the question of how changes in attention affect the rate of acquisition of associations. This property of attention to a $\mathrm{CS}$, relating $\mathrm{CS}$ processing to the rate of associative learning, is often called associability. Typically, some multiplicative relation between the associability of the CS (symbolized as $\alpha$ ) and reinforcement value $(\lambda)$ determines the amount of learning on a conditioning trial $(\Delta V)$; for example, $\Delta V=\alpha \lambda$ (see, e.g., Pearce \& Hall, 1980), or more typically, $\Delta V=\alpha(\lambda-V)$ (e.g., Rescorla \& Wagner, $1972)$, the so-called delta rule, by which the amount of learning to a CS on a trial is proportional to the difference between the amount of learning supportable by the US $(\lambda)$ and the amount already acquired $(V)$. Thus, increased associability $(\alpha)$ of a CS results in more rapid acquisition of associations with that cue, and decreased associability leads to slower learning.

Many early theories, either explicitly or by default, made analogous assumptions about the effects of altered $\alpha$ on performance of CRs. Because performance was some multiplicative function of the amount of attention to a $\mathrm{CS}$ and its associative strength (e.g., $\mathrm{CR}=\alpha V$ ), conditioned responding to a previously trained CS could be enhanced or reduced by subsequent changes in attention to 
that cue. Thus, the extent to which a CS engaged attention determined both the amount of learning that could occur and the magnitude of the CR on any CS presentation.

In contrast, some theories explicitly distinguished between the roles of attentional processing of CSs in the establishment of new learning and in the performance of CRs. Pearce and Hall (1980) distinguished between "controlled" attentional processing, which influences only learning rate or associability, and "automatic" attentional processing of CSs, which somehow bypasses the former mechanism in the generation of performance. In that model (Pearce \& Hall, 1980, pp. 538, 548-549), the amount of learning on any trial is characterized as $\Delta V=s \alpha \lambda$, where $\alpha$ represents a component of associability that varies as a function of conditioning experiences, and $s$ reflects an associability component that is unchanged by experience (and which may be determined instead by intrinsic properties of the CS, like intensity, or the choice of US). Although they fail to describe influences on automatic processing or provide an explicit performance rule, Pearce and Hall (1980, p. 549) are clear in stating that such a rule could not include $\alpha$. Consequently, within that model, changes in controlled attention to a CS affect performance only indirectly, by influencing the value of $V$ attained by any cue. An important implication of this assumption is that substantial changes in the associability of CSs might not be reflected in changes in conditioned responding to that cue, but only in the subsequent acquisition of new associations involving that CS. In much of the research described later in this article, we used procedures that permitted assessment of changes in both a CS's ability to elicit CRs and its ability to participate in new learning.

Theories of attention in conditioning also differ in their statements of the conditions that produce changes in attention. Most of these statements are specifically concerned with experimental situations in which multiple events are in competition for association with a US. The prototypical example is the case of blocking (Kamin, 1968), in which, for example, prior light-US pairings greatly diminish the learning of tone-US associations when a light + tone compound CS is later paired with the US. Attentionbased theories of learning account for this blocking of learning about the tone in terms of how prior learning about one cue may change the allocation of attention to cues added later in training. Although many systems for such allocation have been proposed, a contrast between the proposals of Mackintosh (1975) and Pearce and Hall (1980) is informative.

Mackintosh (1975) proposed that when multiple cues are present on a trial, attention to the best predictor of reinforcement is increased, and attention to poorer predictors is reduced. At the conclusion of each trial, a set of contrasts are made between the outcome received on that trial and the outcome anticipated on the basis of each individual cue present on that trial. By definition, the best predictor of reinforcement is the CS whose associative strength $\left(V_{\mathrm{CS}}\right)$ best matches the reinforcement strength of the US $(\lambda)$. Thus, if $\left|\lambda-V_{\mathrm{CS} 1}\right|<\left|\lambda-V_{\mathrm{CS} 2}\right|$, then $\alpha_{\mathrm{CS} 1}$ will increase and $\alpha_{\mathrm{CS} 2}$ will decrease. In the initial light condi- tioning phase of a blocking experiment, the light is the only predictor of food available, so $\alpha_{\text {light }}$ will increase. When the tone is added to the light, $\left|\lambda-V_{\text {light }}\right|$ will be small, whereas $\left|\lambda-V_{\text {tone }}\right|$ will be large, so $\alpha_{\text {tone }}$ will decrease on each subsequent trial. Consequently, because learning about the tone is a function of both attention and reinforcement, $\Delta V_{\text {tone }}=\alpha_{\text {tone }}\left(\lambda-V_{\text {tone }}\right)$, little conditioning of the tone would be anticipated on subsequent light + tone $\rightarrow$ food pairings.

In contrast, Pearce and Hall (1980) proposed that the associability $(\alpha)$ of a CS decreases as that CS becomes a better predictor of reinforcement and increases only when the CS's predictions are falsified. Although this view at first seems to contradict the intuition embodied in Mackintosh's (1975) theory that it is adaptive to learn to attend to the cues that have proved useful and to learn to ignore the cues that have not, it is important to recall that Pearce and Hall distinguished between the contributions of automatic attentional processing in performance and of controlled processing in learning. Casually speaking, although it may indeed be important that performance be governed by cues that have proved useful in the past, for the purposes of new learning it seems advantageous to engage attention more when confronted with a cue whose significance is unknown than when encountering cues whose consequences are already well established.

Within Pearce and Hall's (1980) theory, $\alpha_{\mathrm{CS}}$ is adjusted after each trial so that it reflects the extent to which the events that followed the CS were well predicted: $\alpha_{\mathrm{CS}}=$ $\lambda-V_{\text {Agg }}$, where $V_{\text {Agg }}$ is the "aggregate prediction" of the US, the sum of the strengths of all cues present on that trial, not just that of the CS in question. The closer $V_{\text {Agg }}$ is to $\lambda$ - that is, the better predicted the US is - the lower will be the value assigned to $\alpha_{\mathrm{CS}}$ after that trial, and the less that will be learned about that CS on subsequent trials (because $\Delta V_{\mathrm{CS}}=s_{\mathrm{CS}} \alpha_{\mathrm{CS}} \lambda$ ). In the blocking experiment, for example, because the US is already well predicted by the light when the tone is first introduced, $\alpha_{\text {tone }}$ will be rapidly adjusted toward zero, minimizing subsequent learning about the tone. However, if an already well-predicted US is omitted, a large mismatch between $V_{\mathrm{Agg}}$ and the $\lambda$ for nonreinforcement (0) would occur; the associability of a CS present on that trial would consequently be adjusted upward again; and subsequent learning about the CS would be enhanced. Put casually, within this theory, the associability of a CS increases whenever the outcome of a conditioning episode is surprising but decreases whenever the outcome is anticipated. The research described in the remainder of this section supports these assumptions about the conditions that produce increases and decreases in associability of CSs and further suggests that different brain systems subserve these incremental and decremental processes.

\section{A. Amygdala-Basal Forebrain Corticopetal System for Incremental, but Not Decremental, Changes in Associability}

In the experiments described in this section, we found that damage to a brain system that includes amygdala $\mathrm{CN}$ prevented increases in CS associability that normally occur 


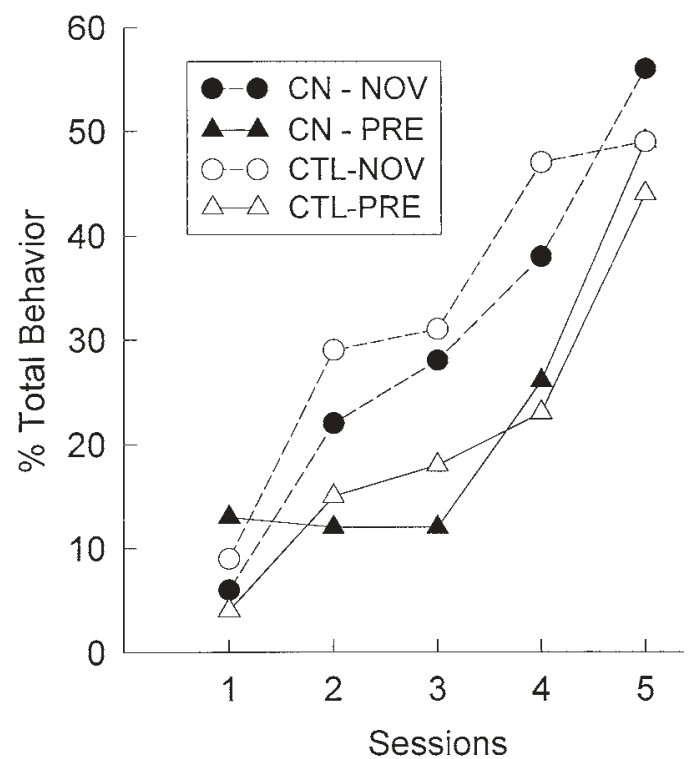

Figure 4. Performance of food cup behavior during two visual conditioned stimuli in the conditioning phase of a latent inhibition experiment reported by Holland and Gallagher (1993a, Experiment 1). The lines labeled $\mathrm{CN}$ refer to performance of rats that had received lesions of the amygdala central nucleus, and those labeled CTL refer to performance of unlesioned control rats. NOV refers to the novel visual cue, and PRE refers to the preexposed visual cue.

when relations between CSs and subsequent events are altered. At the same time, that damage did not affect behavior under circumstances in which decreases in CS associability are presumed to occur. In this section, I will first describe data that show that the most common indicator of reductions in CS associability, the occurrence of latent inhibition, is unaffected by $\mathrm{CN}$ lesions. Next, I will describe data that show that several phenomena thought to reflect incremental changes in CS associability are eliminated by $\mathrm{CN}$ lesions, but that related phenomena attributed to decreases in CS associability are unaffected by those lesions. Finally, I will describe some of our efforts at tracing the brain circuitry involved in incremental changes in CS associability.

Effects of CN lesions on decrements in CS associability: Latent inhibition. In our first experiment here, we investigated the involvement of amygdala $\mathrm{CN}$ in decrements in CS processing by examining the effects of $\mathrm{CN}$ lesions on latent inhibition (Holland \& Gallagher, 1993a,
Experiment 1). In latent inhibition, the acquisition of conditioning to a CS is slowed if that event is presented repeatedly before the conditioning treatment. Presumably, preexposure reduces the associability of the CS, perhaps through some habituation-like process. In our experiment, $\mathrm{CN}-$ lesioned and unlesioned rats received repeated nonreinforced exposure to one of two visual cues. Then, all rats received pairings of each of the two visual cues with food. CN lesions had no effect on latent inhibition (Figure 4). Each control rat and each lesioned rat acquired food cup CRs more rapidly to the novel cue than to the preexposed cue. Thus, $\mathrm{CN}$ damage did not alter performance in a task though to reveal losses of associability of the CS. This conclusion is further supported by the results of experiments, described in the next part of this section, in which other procedures were used to reduce the associability of CS.

$\mathrm{CN}$ lesions and the regulation of associability in serial conditioning. The Pearce-Hall (1980) model clearly predicts that although the associability of a CS should decrease when that CS consistently predicts its outcome, it should be maintained if the $\mathrm{CS}$ is an unreliable predictor of its consequences. For example, partial reinforcement should maintain greater CS associability than should consistent reinforcement. Similarly, CS associability driven down by consistent CS-US pairings should be at least partially restored if the CS is made less predictive. Considerable data from Pearce's laboratory support this claim (e.g., Collins \& Pearce, 1985; Wilson, Boumphrey, \& Pearce, 1992).

In our first experiment in this series (Holland \& Gallagher, 1993a, Experiment 2), we examined the involvement of the amygdala $\mathrm{CN}$ in the augmentation of CS associability that should occur when a consistent predictive relation is shifted to a less predictive relation. We used a serial conditioning procedure described by Wilson et al. (1992), in which the accuracy of a light CS in predicting the occurrence of another CS (a tone) was manipulated, rather than its accuracy in predicting the food US (Table 1). This procedure is somewhat complex, but it provides analytic advantages, which will be described later.

In the first phase of this experiment, lesioned and unlesioned rats were exposed to a serial conditioning procedure in which a light $\rightarrow$ tone sequence was followed by a food US on half the trials (i.e., light $\rightarrow$ tone $\rightarrow$ food and light $\rightarrow$ tone $\rightarrow$ nothing trials). Thus, although the light and tone were both followed by the food US on a $50 \%$ reinforcement schedule, the light was consistently followed by the tone. The tone was expected to acquire a substantial

Table 1

Outline of Procedures for the Wilson,

Boumphrey, and Pearce (1992) Task

\begin{tabular}{cllc}
\hline Group & \multicolumn{1}{c}{ Phase 1 } & \multicolumn{1}{c}{ Phase 2 } & \multicolumn{1}{c}{ Test } \\
\hline Consistent & light $\rightarrow$ tone $\rightarrow$ food & light $\rightarrow$ tone $\rightarrow$ food & light $\rightarrow$ food \\
Shift & light $\rightarrow$ tone $\rightarrow$ nothing & light $\rightarrow$ tone $\rightarrow$ nothing & \\
& $\begin{array}{l}\text { light } \rightarrow \text { tone } \rightarrow \text { food } \\
\text { light } \rightarrow \text { tone } \rightarrow \text { nothing }\end{array}$ & $\begin{array}{l}\text { light } \rightarrow \text { tone } \rightarrow \text { food } \\
\text { light } \rightarrow \text { nothing }\end{array}$ & light $\rightarrow$ food \\
&
\end{tabular}

Note-Entries in italics denote the only difference in the treatments received by subjects in the consistent and shift treatments. 
CN Lesion Groups

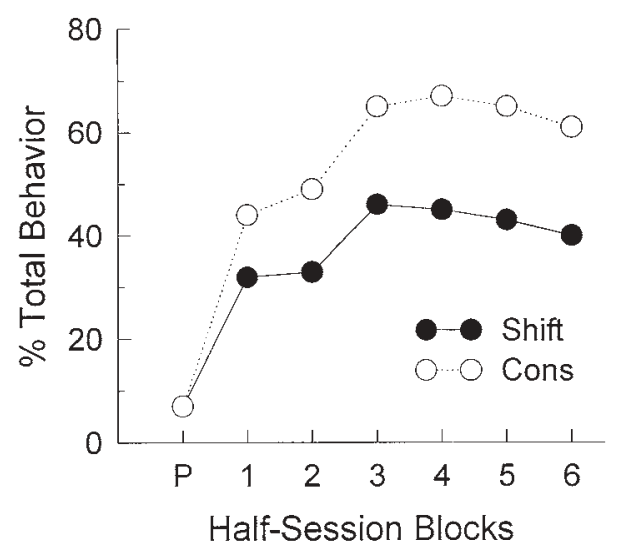

Control Groups

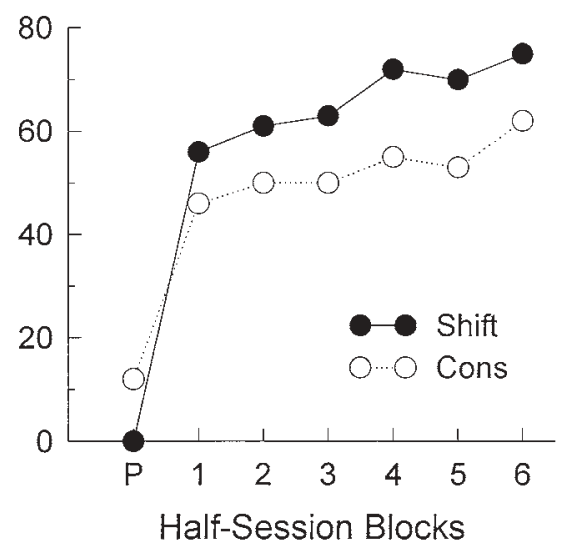

Figure 5. Performance of food cup behavior during a light conditioned stimulus in the test phase of an experiment reported by Holland and Gallagher (1993a, Experiment 2). Prior to this test, some rats received a shift in the predictive relation between that light and a tone, whereas for the other rats, a consistent (Cons) relation was maintained. The left panel shows responding of rats that received lesions of the amygdala central nucleus $(\mathrm{CN})$, and the right panel shows responding of unlesioned control rats. $P$ on the abscissae refers to responding during the light on the last session of the previous phase.

CR as a consequence of this schedule, but because of its relatively poor temporal relation with food, and because of its intrinsically low salience $(s)$, the light was expected to acquire only a minimal CR. Most important for our purposes, the associability of the light should decline, because the light would come to accurately predict the tone as serial conditioning proceeded.

In the second phase, one group of lesioned (CN-CONS) and one group of unlesioned rats (CTL-CONS) continued to receive the Phase 1 procedures, light $\rightarrow$ tone $\rightarrow$ food and light $\rightarrow$ tone $\rightarrow$ nothing trials. This was the consistent procedure. In a second pair of lesioned (CN-SHIFT) and unlesioned (CTL-SHIFT) groups, the light $\rightarrow$ tone $\rightarrow$ nothing trials were replaced by light-alone trials. Thus, for the second pair of groups, the light's predictive value was shifted: Although its relation with the food US was unchanged, the light no longer reliably predicted the tone. As a result, the associability of the light should increase.

We assessed changes in the light's associability by pairing the light directly with food in a final test phase. To the extent that the Phase 2 shift in the light's predictive value for Groups CN-SHIFT and CTL-SHIFT increased the light's associability, light-food conditioning in the test phase should occur more rapidly in those groups than in the CONS groups. However, to the extent that such an increment in attention was mediated by $\mathrm{CN}$, enhanced conditioning would be observed only in unlesioned rats (Group CTLSHIFT), and not in lesioned rats (Group CN-SHIFT).

Figure 5 shows the results of that test phase. First, consistent with the findings of Wilson et al. (1992), among the unlesioned CTL rats, those that received a shift in the predictive accuracy of the light in Phase 2 (Group CTLSHIFT) showed more food cup responding than did those that did not (Group CTL-CON). Thus, the shift in the light's predictive value apparently enhanced the light's as- sociability in intact rats. In contrast, in the lesioned rats, food cup responding was lower in the shifted rats (Group CN-SHIFT) than in the unshifted rats (Group CN-CONS). Clearly, among these rats, shifting the light's predictive accuracy did not confer an advantage, so we concluded that this shift did not produce incremental changes in the light's associability in rats with $\mathrm{CN}$ damage. Furthermore, the comparable test performance of the lesioned and unlesioned rats in the consistent (CONS) condition suggests that any losses in the light's associability (which are presumed to occur with that training procedure) were not affected by the lesions. Thus, the results of this experiment implicate the amygdala CN in increases in a CS's associability when its predictive validity is reduced, but not in decreases in a CS's associability when it is a consistent predictor of its consequences.

The observation in lesioned rats of significantly less test responding after the shift procedure than after the consistent procedure is also interesting. In the absence of a mechanism for increasing associability when predictive validity is reduced, there are several reasons to expect that the acquisition of light-food learning would be greater after exposure to the consistent treatment as opposed to the shift treatment. First, without this process for increasing CS associability, the shift procedure might be particularly effective at decreasing the light's associability. Most data (see, e.g., Lubow, Wagner, \& Weiner, 1982) show that reductions in associability of a CS in latent inhibition procedures are greater when the CS is preexposed by itself than when it is followed by another cue. In our studies, note that whereas the shift procedure contains both lightalone and light $\rightarrow$ tone trials, the consistent procedure contains only light $\rightarrow$ tone trials.

Similarly, most theories of conditioning would predict that the shift procedure would establish weaker light-food 
Table 2

Outline of Procedures for Holland's (1988)

Blocking and Unblocking Tasks

\begin{tabular}{lllr}
\hline Group & \multicolumn{1}{c}{ Phase 1} & \multicolumn{1}{c}{ Phase 2} & Test \\
\hline CON & $\mathrm{fd} 1 \rightarrow \mathrm{fd} 2$ & light + tone $\rightarrow \mathrm{fd} 1 \rightarrow \mathrm{fd} 2$ & tone? \\
HI & light $\rightarrow \mathrm{fd} 1 \rightarrow \mathrm{fd} 2$ & light + tone $\rightarrow \mathrm{fd} 1 \rightarrow \mathrm{fd} 2$ & tone? \\
LO & light $\rightarrow \mathrm{fd} 1$ & light + tone $\rightarrow \mathrm{fd} 1$ & tone? \\
UP & light $\rightarrow \mathrm{fd} 1$ & light + tone $\rightarrow \mathrm{fd} 1 \rightarrow \mathrm{fd} 2$ & tone? \\
DN & light $\rightarrow \mathrm{fd} 1 \rightarrow \mathrm{fd} 2$ & light + tone $\rightarrow \mathrm{fd} 1$ & tone? \\
\hline
\end{tabular}

Note - fd 1 was the delivery of one $45-\mathrm{mg}$ food pellet; fd 2 was the delivery of two 45 -mg pellets, $5 \mathrm{sec}$ after fd 1 .

associations than would the consistent procedure. In the consistent procedure, the effects of both reinforced and nonreinforced trials would be divided between the light and tone cues. In contrast, in the shift procedure, the effects of reinforced trials would again be divided between light and tone, but the light would absorb all of the effects of nonreinforced trials. Although our choices of event salience and time intervals in the serial conditioning procedures were intended to minimize conditioning to the light, and at the end of the second phase of the experiment there was little evidence for CRs to that cue, it is plausible that subthreshold light-food associations were formed before the test phase. Indeed, the very rapid emergence of responding to the light in the test is consistent with the prior acquisition of some light-food association.

Thus, there is ample reason to believe that the shift procedure would produce less responding in the test phase than would the consistent procedure, except for the operation of an associability-enhancing mechanism. Because (we posit), $\mathrm{CN}$ lesions interfere with such a mechanism, the superiority of the performance of $\mathrm{CN}$-lesioned rats trained with the consistent procedure (Group $\mathrm{CN}-\mathrm{CONS}$ ) to that of rats trained with the shift procedure (Group CNSHIFT) simply reveals the normal operation of other processes that reduce associability and/or associative strength. The CN-lesioned rat might then be viewed as a reduced preparation, which could prove useful for investigations of these other processes, unencumbered by incremental changes in associability.

Finally, because in this serial conditioning procedure the predictive relations between two CSs (rather than between CS and US) were altered, a variety of less interesting accounts of the results may be excluded. For example, because the cue of interest (the light) maintains the same partial reinforcement relation with the food US in all conditions, any effects of $\mathrm{CN}$ damage on partial reinforcement effects, processing of reward and nonreward, frustrative nonreward, and so forth are controlled. These issues are of particular importance to investigations of amygdala function, because it has often been claimed that the amygdala underlies some of those processes (e.g., by Henke \& Maxwell, 1973; Kemble \& Beckman, 1970).

$\mathrm{CN}$ lesions and the regulation of associability in blocking and unblocking. In a second attempt to examine the effects of $\mathrm{CN}$ lesions on increments and decrements in the associability of CSs, we used procedures of blocking and unblocking. In blocking, prior training of one element (A) of a compound stimulus (AX) interferes with conditioning of another element $(\mathrm{X})$ when the compound is paired with the same reinforcer as that which has been used in training of the first element (Kamin,1968). However, if A's prior training involves a reinforcer different (usually in magnitude or quantity) from the one later paired with the AX compound, blocking is substantially reduced (unblocking); that is, $\mathrm{X}$ often acquires conditioning. Within Pearce and Hall's (1980) theory, blocking occurs because the associability of $\mathrm{X}$ is rapidly driven lower because the US is already well predicted by A. On the other hand, in the unblocking procedure, the shift in the US when X is introduced slows the loss of X's associability because the new US is not well predicted by A. Consequently, pairings of AX with the new US result in significant conditioning to $\mathrm{X}$. Interestingly, within this theory, unblocking should occur whether the US is underpredicted (increased in magnitude) or overpredicted (decreased in magnitude). In support of this prediction, the paradoxical outcome that reducing the magnitude of the US when $\mathrm{X}$ is added enhances conditioning to the tone has been observed frequently in intact rats (Dickinson, Hall, \& Mackintosh, 1976; Holland, 1984b, 1988).

Holland and Gallagher (1993b, Experiment 1) examined the effects of $\mathrm{CN}$ lesions on both blocking and unblocking. If $\mathrm{CN}$ lesions interfere with a mechanism responsible for increments in CS associability, but not one responsible for decrements, then, according to Pearce and Hall (1980), lesioned rats should show normal blocking but fail to exhibit unblocking.

Table 2 provides a summary of the design of this experiment. In the first phase, two groups (HI and DN) of $\mathrm{CN}$-lesioned and unlesioned control rats received pairings of a visual (light) $\mathrm{CS}$ with the delivery of a multiple-pellet food reinforcer (one pellet followed $5 \mathrm{sec}$ later by two pellets), and two groups (LO and UP) received pairings of the light CS with a single-pellet US. A fifth group (CON), again composed of both $\mathrm{CN}$-lesioned and intact control rats, received unsignaled deliveries of the multiple-pellet US. Next, all groups received training with a compound of the light and a novel tone. In Groups CON, HI, and UP, the light + tone compound was paired with the multiple-pellet reinforcer, and in Groups LO and DN, it was paired with the single-pellet reinforcer. Finally, all groups received a test of responding to the tone alone. 


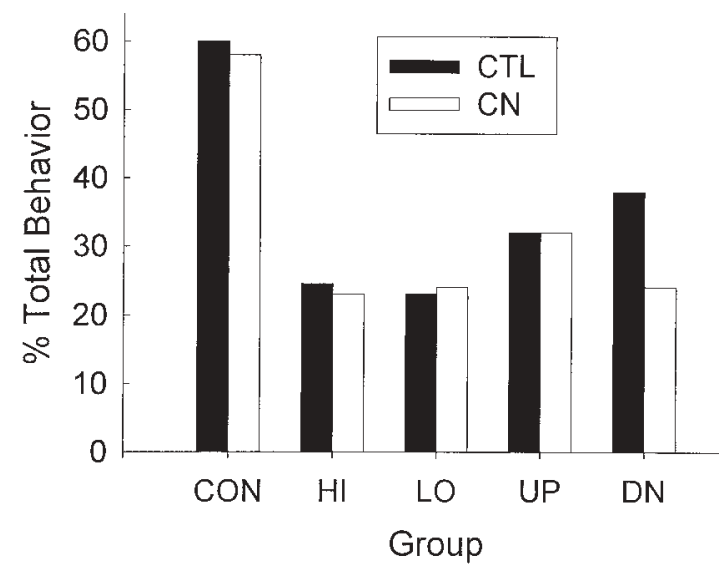

Figure 6. Performance of food cup behavior during a nonreinforced test of a tone conditioned stimulus after blocking and unblocking procedures of an experiment reported by Holland and Gallagher (1993b, Experiment 1). The treatments received by the five groups are described in the text. The bars labeled $\mathrm{CN}$ refer to the performance of rats that had received lesions of the amygdala central nucleus, and the bars labeled CTL refer to the performance of unlesioned control rats.

Comparison of Groups HI and CON provided the standard blocking comparison: both received light + tone-US pairings, but only group $\mathrm{HI}$ received prior training of the light. Unblocking was evaluated by comparing the performance of Groups UP and DN with that of Groups HI and LO: At the start of compound training, the US was shifted up from one pellet to multiple pellets in Group UP and down from multiple pellets to one pellet in Group DN, but was unchanged in Groups HI and LO.

Figure 6 shows the results of the final test of the tone CS. First, prior training of the light blocked learning to the tone equally well in lesioned and control rats, which is consistent with our previous evidence that the amygdala $\mathrm{CN}$ is not importantly involved in decremental changes in attention that occur when USs are well predicted. There was little responding in Group HI, which received lightmultiple-pellet US pairings prior to light + tone-multiplepellet US pairings, in either lesioned or intact rats. In contrast, there was substantial and equivalent responding in both the lesioned and unlesioned rats in Group CON, which received no training of the light prior to pairings of the light + tone compound with the multiple-pellet US.

Second, consistent with the results of previous experiments done with these procedures (Holland, 1984b, 1988) was the finding that the intact rats showed reliable unblocking with both upward and downward shifts in the US. Responding to the tone in the test was greater in the unlesioned rats in both Group UP and Group DN, compared with the responding of the unlesioned rats in Groups HI and LO, which received the same US in both phases. Third, although CN-lesioned rats in Group UP also showed unblocking, CN-lesioned rats in Group DN showed no evidence of unblocking when the US's value was shifted downward: The performance of the lesioned rats in that group was reliably lower than the performance of the unlesioned rats in that group, and it was no different from that of the lesioned rats in Groups HI and LO. Thus, although the failure to observe an effect of the $\mathrm{CN}$ lesions in Group UP is troublesome at first glance, the abolition of unblocking with downshifts in US value after $\mathrm{CN}$ lesions in Group DN is consistent with our hypothesis that increases in associability produced by disconfirmation of US expectancies would be absent in rats with $\mathrm{CN}$ damage.

It is important to note that other evidence from this unblocking experiment showed that the shift in value of the US was detected by the $\mathrm{CN}$-lesioned rats. Both lesioned and intact rats exhibited comparable learning to the original light CS when it was paired with the larger US in the first phase $(83.3 \%$ and $79.2 \%$ responding, respectively, on the final session of that phase) and showed comparable losses in responding when the compound cue was paired with the single-pellet US in the second phase $(65.3 \%$ and $68.8 \%$ responding, respectively, on the final day). Thus, the failure of $\mathrm{CN}$-lesioned rats to show unblocking is probably not attributable to deficits in detecting differences in US magnitude (sometimes found in rats with large, unspecific damage to the amygdala; see, e.g., Kemble \& Beckman, 1970), but is more likely the consequence of their inability to increase attention to the tone when the CS-reinforcement relation was altered.

Our failure to observe reliable $\mathrm{CN}$ lesion effects on unblocking with upshifts in US value, replicated in a subsequent experiment (Holland \& Gallagher, 1993b, Experiment 2), is interesting in its own right. One possible account for this finding is that the necessary condition for engaging the incremental process is not merely a discrepancy between anticipated and actual outcomes, but instead a negative discrepancy: Increments in associability may occur only when things get worse. One version of such a view has historical precedent in the study of the effects of frustrative nonreward: For example, Henke and Maxwell (1973) claimed that amygdala damage made rats less subject to the effects of frustrative nonreward. If it is assumed that unblocking with downshifts is the consequence of enhanced responding due to frustrative nonreward (and not alterations in associability), then our observation of $\mathrm{CN}$ lesion-induced deficits in unblocking with downshifts but not upshifts in US value is easily explained. Although this possibility cannot be ruled out, two observations make it less likely. First, Holland and Gallagher (1993b) and Holland $(1984 \mathrm{~b}, 1988)$ presented evidence that unblocking in this situation is not the consequence of frustrative nonreward. Second, comparable CN lesion effects were observed with the Wilson et al. (1992) procedure, which minimized the potential contributions of frustrative nonreward (Holland \& Gallagher, 1993a; see earlier discussion). Of course, ruling out frustrative nonreward as the mediator of the lesion effects that we have observed does not rule out the more general claim that increments in associability are enhanced only with negative expectancy-outcome discrepancies. At this point, we have no further evidence bearing on this question. 
Holland and Gallagher (1993b) favored a second account for their failure to find $\mathrm{CN}$ lesion effects on unblocking when a larger US was substituted for a smaller one (Group UP). They noted that some conditioning of the added tone element in Group UP would be anticipated even without an associability-enhancing process, simply because the magnitude of the US was increased when the tone was introduced. For example, within the RescorlaWagner (1972) model, the difference between the amount of conditioning supportable by the multiple-pellet US and that already conditioned to the light as a result of its pairings with the single-pellet US would be split between the light and tone, proportional to their saliences. Thus, the unblocking observed in Group UP might reflect conditioning processes independent of the enhancement of associability, and hence immune to the effects of $\mathrm{CN}$ lesions.

Of course, the same logic that allows us to attribute the unblocking observed in Group UP to processes other than the enhancement of associability also forces us to reexamine our claim that the failure of $\mathrm{CN}$ lesions to interfere with blocking indicates that $\mathrm{CN}$ is not involved in an associability-reducing mechanism. The blocking observed in this study may not have involved reductions in X's associability at all but instead may have been produced by reinforcement mechanisms. For example, within the Rescorla-Wagner (1972) model, A's prediction of the US interferes with conditioning of $X$ by rendering that US ineffective as a reinforcer, rather than by reducing X's associability. Thus, even if $\mathrm{CN}$ lesions indeed resulted in the maintenance of X's associability, blocking would still occur because the US was made ineffective: With no effective US, X's associability would be irrelevant.

We addressed this problem by exploiting a hybrid procedure that combines elements of both blocking and unblocking, described first by Mackintosh and Turner (1971) and studied in this conditioning preparation by Holland (1985). CN-lesioned and unlesioned control rats were first given pairings of a light CS with either the single- or the multiple-pellet reinforcer used in the previous experiment. Later, all rats received pairings of a light + tone compound with the other US, followed by a test of responding to the tone alone. Thus, half the rats received upshifts and half received downshifts in US value. However, half the rats also received an intervening phase in which the light + tone compound was paired with the same US as was used to train the light. Thus, these subjects (Groups B-UP and B$\mathrm{DN}$ ) received exposure to a standard blocking treatment, followed by unblocking with either upshifts or downshifts in US value. The remaining rats (Groups N-UP and NDN) received no explicit treatment during this intervening blocking phase and thus received only unblocking treatment, with either upshifts or downshifts in US value.

The major purpose of this study was to examine the nature of changes that occurred in the intervening blocking phase. If blocking is solely the consequence of reinforcement effects, as specified by the Rescorla-Wagner model, the intervening blocking phase should not affect subsequent unblocking: The added tone should acquire no con- ditioning in the blocking phase, but no changes in its associability would be expected either. In contrast, to the extent that blocking reflects a decrease in X's associability, the rats that received the intervening blocking phase should start the unblocking phase with an X of substantially lower associability than should the other rats. Consequently, the effects of the unblocking treatment on conditioning to $\mathrm{X}$ should be reduced, relative to performance of the rats that did not experience the intervening blocking treatment. Thus, among intact rats, the observation of more conditioning in Groups N-UP and N-DN than in Groups B-UP and B-DN would indicate that the blocking phase had indeed reduced the associability of X. Holland (1985) noted just that outcome. If $\mathrm{CN}$ lesions interfered with losses in CS associability, CN-lesioned rats should be less affected by the intervening blocking phase than intact rats, whereas if $\mathrm{CN}$ lesions do not affect decreases in CS associability, as we hypothesized, lesioned and intact rats should be similarly affected by the intervening blocking phase.

Figure 7 shows the results of the final test phase in this study. First, consistent with Holland's (1985) findings was the finding that the intact control rats in Groups B-UP and B-DN showed significantly lower responding to the tone than did those in Groups N-UP and N-DN. Thus, the intervening blocking procedure lowered the associability of $\mathrm{X}$ in the former groups. Second, CN-lesioned rats in Groups B-UP and N-UP showed responding identical to that in the intact rats in the irrespective groups, suggesting that the intervening blocking procedure reduced X's associability

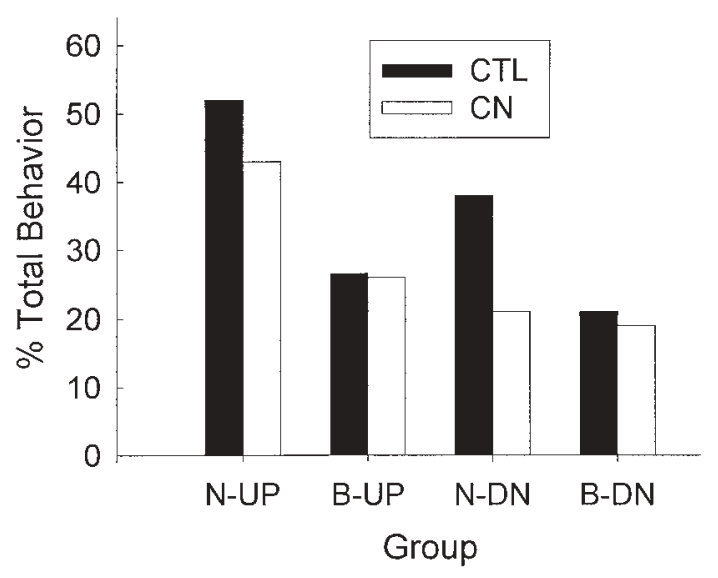

Figure 7. Performance of food cup behavior during a nonreinforced test of a tone conditioned stimulus after unblocking procedures in an experiment reported by Holland and Gallagher (1993b, Experiment 2). The rats received either increases (UP) or decreases (DN) in the value of the reinforcer presented after a light + tone compound stimulus in an unblocking phase immediately prior to this test. Prior to the unblocking phase, but after initial light-food pairings, the rats in the groups labeled $B$ received pairings of the light + tone compound with the food reinforcer received in the initial light-food training, whereas the rats in groups labeled DN did not. The bars labeled $\mathrm{CN}$ refer to the performance of rats that had received lesions of the amygdala central nucleus, and the bars labeled CTL refer to the performance of unlesioned control rats. 
in CN-lesioned rats equally well. Third, there was little evidence for responding in the $\mathrm{CN}$-lesioned rats in either Group B-DN or Group N-DN. This outcome is consistent with the results of the experiment just described, in which unblocking with downshifts was not observed in rats with CN lesions.

In summary, the results of this experiment replicated those of the previous experiment in showing that $\mathrm{CN}$ lesions interfered with the occurrence of unblocking when the US value was shifted down, but not when the US value was shifted up. In addition, they supported our claim that the blocking procedures used in the previous experiment (and in this experiment) reduced the associability of the added tone CS, and further, that this reduction in CS associability was not affected by $\mathrm{CN}$ lesions.

Use of CN-lesioned rats to identify the contribution of increments in associability to behavioral performance. The strategy in the previous experiments was to determine the nature of $\mathrm{CN}$ function by examining the effects of $\mathrm{CN}$ lesions on performance in tasks already believed, on the basis of prior behavioral investigation, to involve incremental changes in CS associability. A complementary strategy, whereby $\mathrm{CN}$-lesioned rats are used to provide evidence that incremental changes in associability are involved in performance on some poorly understood behavioral task, may also prove useful. If some performance is hypothesized to occur because of enhanced CS associability, CN-lesioned rats should fail to exhibit that performance. Engaging in a bit of bootstrapping, observation of such failure implicates enhanced associability in the normal performance of the task. Lack of an effect of $\mathrm{CN}$ lesions on the phenomenon would make it less likely that it was the consequence of alterations in CS processing.

Recently, Lindsey Ciali, Michael Conley, Derick Davis, Jennifer Thornton, and I used such a bootstrapping strategy to explore a puzzling finding reported by Rescorla (1991). In Rescorla's (1991) study, one group of pigeons was trained in a serial feature negative discrimination task in which one cue was reinforced when it was presented alone, and it was nonreinforced when it was preceded by another cue $(\mathrm{A}+, \mathrm{X} \rightarrow \mathrm{A}-)$. Another group of pigeons was trained with a serial negative patterning discrimination $(\mathrm{A}+, \mathrm{X}+, \mathrm{X} \rightarrow \mathrm{A}-)$ in which each of the two elements was separately reinforced, but nonreinforced when they were presented in compound. Most accounts of discrimination learning predict that the latter task would be more difficult than the former task: although the feature-negative discrimination could be solved by X's acquisition of inhibitory tendencies, the negative patterning discrimination could not be. However, Rescorla (1991) found that pigeons trained with the serial negative patterning discrimination learned more rapidly. He attributed this finding to the induction of increases in X's associability because of its pairings with food on some trials, as might be anticipated within a theory like Mackintosh's (1975). From the perspective of Pearce and Hall (1980), the serial negative patterning procedure may maintain X's associability because the subjects' expectancies are violated on both $\mathrm{X}+$ trials (A is omitted but the US is presented) and $\mathrm{X} \rightarrow \mathrm{A}-$ trials ( $\mathrm{A}$ is presented but the US is omitted).

We reasoned that if this phenomenon was indeed the consequence of enhanced associability of $\mathrm{X}$ in the negative patterning (NP) group relative to the feature negative (FN) group, the advantage of the patterning procedure should disappear in CN-lesioned rats. In our (unpublished) study, $\mathrm{CN}$-lesioned and unlesioned control rats were given training on Pavlovian serial feature negative (tone + , light $\rightarrow$ tone-) or serial negative patterning discriminations (tone + , light + , light $\rightarrow$ tone -$)$. Although control rats showed reliably faster acquisition when trained with the patterning task (consistent with Rescorla's data), lesioned rats showed no such superiority (Figure 8). This pattern of data is consistent with the claim that the superiority of learning of the patterning task to learning of the feature negative task was the result of a $\mathrm{CN}$-dependent associability-enhancing mechanism in the intact rats.

One might argue that such a mechanism should also be engaged with the serial feature negative procedure, although perhaps to a lesser extent, because on $\mathrm{X} \rightarrow \mathrm{A}-$ trials, the expected US is omitted. The lack of an effect of CN lesions on performance on the serial feature negative task, however, may reflect the relatively lengthy temporal interval between the presentation of $\mathrm{X}$ and the omission of the US at the end of A. Note that for subjects in the patterning condition, an expectancy of the US based on X-US associations is disconfirmed immediately after $\mathrm{X}$ on $\mathrm{X} \rightarrow \mathrm{A}-$ trials and so should have greater impact on X's associability (see Collins \& Pearce, 1985, for a related point).

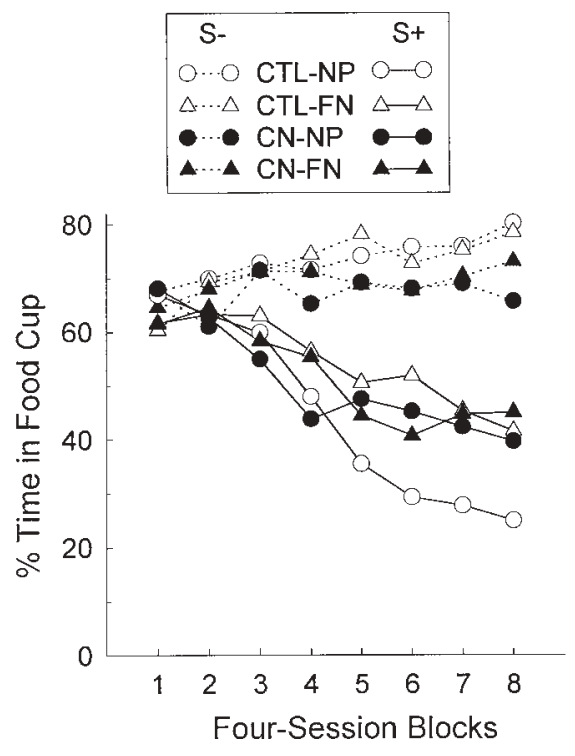

Figure 8. Performance on serial negative patterning (NP) and serial feature negative (FN) discriminations of rats with lesions of the amygdala central nucleus $(\mathrm{CN})$ and of unlesioned control rats (CTL). S+, responding on reinforced target-alone trials; $S-$, responding during the target on nonreinforced compound trials. Time in the food cup was measured automatically by a photocell circuit. 

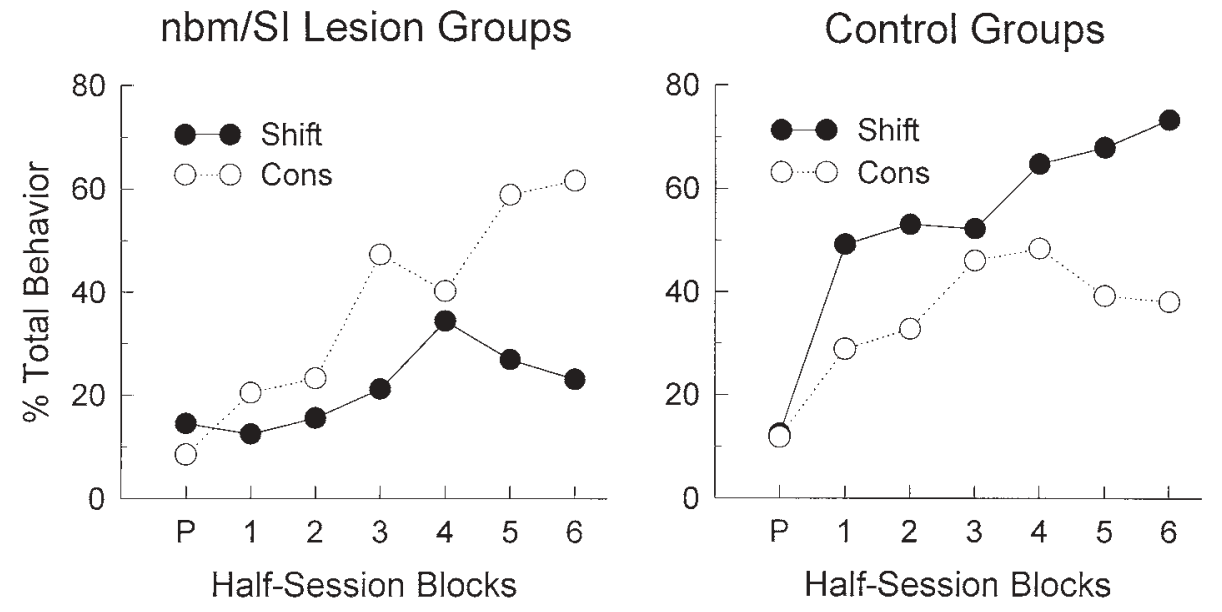

Figure 9. Performance of food cup behavior during a light conditioned stimulus in the test phase of an experiment reported by Chiba, Bucci, Holland, and Gallagher (1995, Experiment 1). Prior to this test, some rats received a shift in the predictive relation between that light and a tone, whereas for the other rats, a consistent (Cons) relation was maintained. The left panel shows responding of rats that received lesions of the nucleus basalis/substantia innominata $(\mathrm{nbm} / \mathrm{SI})$, and the right panel shows the responding of unlesioned control rats. $P$ on the abscissae refers to responding during the light on the last session of the previous phase.

Of course, $\mathrm{CN}$ lesions might have affected performance in these tasks in other ways instead. For example, one might argue that $\mathrm{CN}$ lesions affect configural learning and that only the patterning task involves configural learning. However, unlike this account (for example), the bootstrapping argument just described is consistent both with the superiority of the patterning procedure in intact rats and with other known effects of the amygdala $\mathrm{CN}$ lesion.

Higher brain systems for increases in associability. We have used the various conditioning procedures just described to help us trace the brain pathways used in the generation of increments in CS associability. Most of these studies have relied on the procedure of Wilson et al. (1992), which we have found to generate robust data. Our strategy has been to use the results of anatomical and metabolic mapping studies (e.g., Bucci, Chiba, Holland, \& Gallagher, 1995) to establish a plausible set of brain pathways to investigate, and then to examine the effects of lesions at various levels in these pathways on performance in these tasks. Although these studies were primarily intended to provide information about the flow of information in the brain, some have also yielded data interesting from a purely behavioral perspective.

Of special interest for our research is the extensive $\mathrm{CN}$ output to systems in the basal forebrain, which in turn regulate cortical processing. In rats, a system of large cholinergic neurons, scattered throughout the sublenticular substantia innominata (SI), ventral globus pallidus, and nucleus basalis (nbm), also receives substantial input from the $\mathrm{CN}$ (Grove, 1988). Recent studies indicate that the basal forebrain is involved in the allocation of attentional resources in multiple-choice reaction time experiments (e.g., Muir,
Dunnett, Robbins, \& Everitt, 1992) and that the amygdala $\mathrm{CN}$ regulates activity of the projection from basal forebrain to cortical regions (Kapp, Supple, \& Whalen, 1994). Our research suggests further that this circuitry is involved in incremental changes in CS processing during conditioning.

Nucleus basalis/substantia innominata $(\mathrm{nbm} / \mathrm{SI})$ lesions and the regulation of associability in serial conditioning. If the CN's mediation of variations in attentional processing involves this circuitry, destruction of the cholinergic neurons in the $\mathrm{nbm} / \mathrm{SI}$ should produce effects similar to those of $\mathrm{CN}$ lesions. In this experiment (Chiba, Bucci, Holland, \& Gallagher, 1995, Experiment 1), we used an immunotoxin ( ${ }^{192} \mathrm{IgG}$-saporin) to selectively lesion the cholinergic component of the magnocellular corticopetal system in the nbm/SI; intermingled noncholinergic neurons in this region were undamaged.

$\mathrm{Nbm} / \mathrm{SI}$-lesioned and unlesioned rats received training on the Wilson et al. (1992) task, with treatments identical to those described earlier (Table 1). Figure 9 shows the results of the final test phase of this experiment. As can be seen by comparing Figures 5 and 9, the effects of nbm/SI lesions and $\mathrm{CN}$ lesions were virtually identical. Among the unlesioned rats, subjects in the SHIFT condition showed more responding than did those in the consistent condition, but among the lesioned rats, SHIFT subjects showed less responding. Furthermore, the lesions did not affect the performance of rats in the consistent condition. Thus, both $\mathrm{CN}$ and $\mathrm{nbm} / \mathrm{SI}$ lesions disrupted incremental changes in CS processing when the predictive validity of the CS was altered, but did not affect losses in CS processing presumed to occur when the CS was a consistent predictor of subsequent events. 


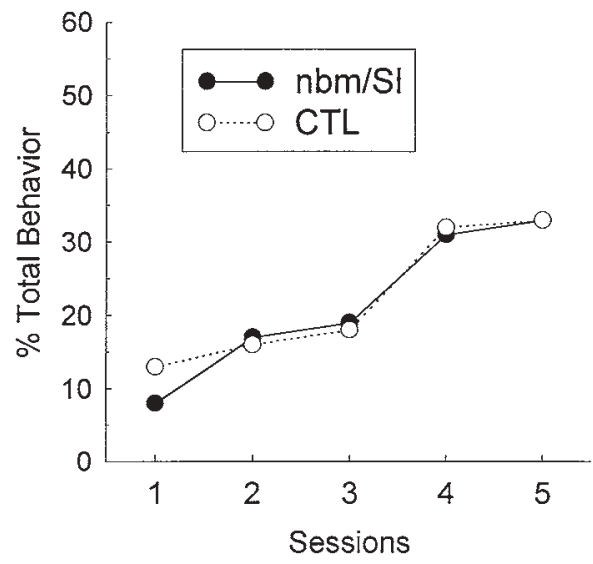

Figure 10. Performance of rear behavior during light-food pairings in an experiment reported by Chiba, Bucci, Holland, and Gallagher (1995, Experiment 1). The line labeled nbm/SI shows responding of rats that received lesions of nucleus basalis/substantia innominata and the line labeled CTL shows responding of unlesioned control rats.

More direct evidence that this circuitry is not an important contributor to decremental changes in associability was provided by the results of another experiment in this series (Chiba et al., 1995, Experiment 2). In that experiment, using procedures identical to those used by Holland and Gallagher (1993a) in their examination of the effects of $\mathrm{CN}$ lesions on latent inhibition, we found no effects of similar immunotoxic lesions of the $\mathrm{nbm} / \mathrm{SI}$ on latent inhibition.

Significantly, however, there was one major difference between the effects of $\mathrm{CN}$ and $\mathrm{SI} / \mathrm{nbm}$ lesions in these two experiments. The acquisition of conditioned orienting (rearing to the visual cue) in the test phase, which was prevented by the $\mathrm{CN}$ lesion, was unaffected by the $\mathrm{nbm} / \mathrm{SI}$ lesion (Figure 10). Thus, the extra-amygdalar circuitry for enhanced CS associability and enhanced orienting is apparently quite different. Whereas a pathway incorporating $\mathrm{CN}$ and $\mathrm{nbm} / \mathrm{SI}$ is critical to increments in CS associability, conditioned orienting is apparently mediated by the $\mathrm{CN}$-nigrostriatal path described earlier.

Other evidence also supports the possibility that these two behavioral functions of the $\mathrm{CN}$ are separable. For example, although Han et al. (1997) found that unilateral lesions of the $\mathrm{CN}$ (unlike bilateral lesions of the $\mathrm{CN}$ ) have no deleterious effects on conditioned orienting, Han, Gallagher, and Holland (unpublished data) found that unilateral CN lesions partially disrupted performance in the Wilson et al. (1992) task. In that study, unilaterally lesioned rats exposed to the shift and consistent conditions displayed similar performance in the test phase. From a behavioral perspective, it is not surprising that increased associability and conditioned orienting are not subserved by the same neural circuitry, because changes in conditioned orienting and putative changes in associability are often uncorrelated. For example, within Pearce and Hall's (1980) theory, the associability of a CS consistently paired with the US should decrease continuously, whereas conditioned
ORs in both my and Gallagher's laboratories are maintained throughout conditioning. Similarly, despite the presumed losses and increments in the associability of the initial light cue over the course of our serial conditioning experiments done with the Wilson et al. task, conditioned orienting did not appear until the final test phase, when the light was placed in a temporally effective conditioning relation with food.

It is important to recognize that Pearce's lab has reported a very different pattern of orienting behavior, consistent with the putative changes in associability (e.g., Kaye \& Pearce, 1984, and Wilson et al., 1992). However, the topography of the orienting behavior that they have observed in the presence of visual cues differs from that which we report. Their orienting response involves touching or looking at the source of a small, localizable light source placed at approximately head level. In contrast, our OR, elicited by a more diffuse light source above the chamber, includes only rear behavior, whatever its orientation. We have not observed the behavior described by Kaye and Pearce (1984) in our preparation. It would be very interesting to determine whether the OR that they report, unlike ours, would be affected by lesions of the SI and hence more directly related to brain mechanisms for associability changes, as they claim.

Posterior parietal cortex and enhanced associability. The large cholinergic cells in the SI destroyed in our lesions have widespread cortical projections. Recent unpublished work in our laboratories by David Bucci suggests that projections of these neurons to the posterior parietal cortex, a region bordering the somatosensory and visual cortex but possessing strong multimodal input, are critical to the increases in associability observed in the serial conditioning task. Bucci was led to examine the effects of disrupting this pathway for two reasons. First, considerable research has shown that humans' performance on tasks that demand shifts in spatial attention is disrupted in patients with lesions in this area (Peterson, Robinson, \& Currie, 1989; Posner, Walker, Friedrich, \& Rafal, 1984; Salzmann, 1995). Similarly, in analogous tasks with monkeys, Salzmann found correlated electrical activity in both the posterior parietal cortex and the pulvinar, a main innervator of the former region.

Second, this region of the rat's cortex was implicated by the results of a mapping study done with the Wilson et al. (1992) task (Bucci et al., 1995). In Bucci et al.'s study, intact rats were given Phase 1 training on the Wilson et al. task, the same as in our previous studies. However, the rats were given only two sessions of Phase 2 treatment with the shift or consistent procedures. Half the rats were then given light-food pairings, to assess the behavioral effects of this abbreviated Phase 2 training. These rats showed the usual shift condition superiority. The other half of the rats were sacrificed immediately after the second Phase 2 training session, and assays of the activity of $c$-fos, an immediate early gene product widely used as an indicator of brain metabolic activity, were performed. The idea was that CS associability should be adjusted soon after the 
PPC Lesion Groups

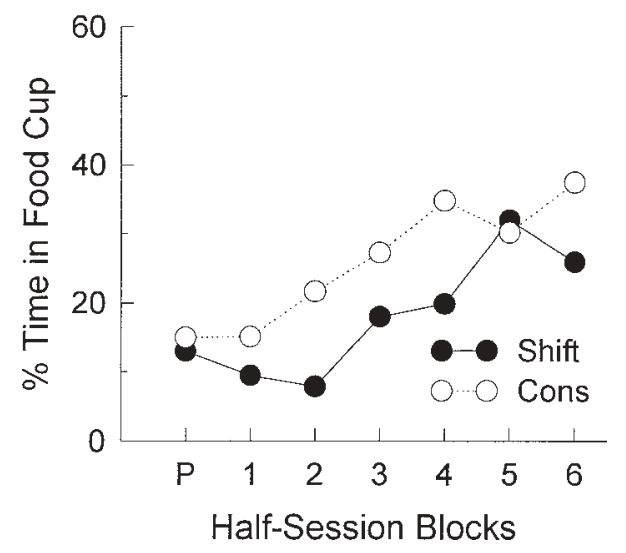

Control Groups

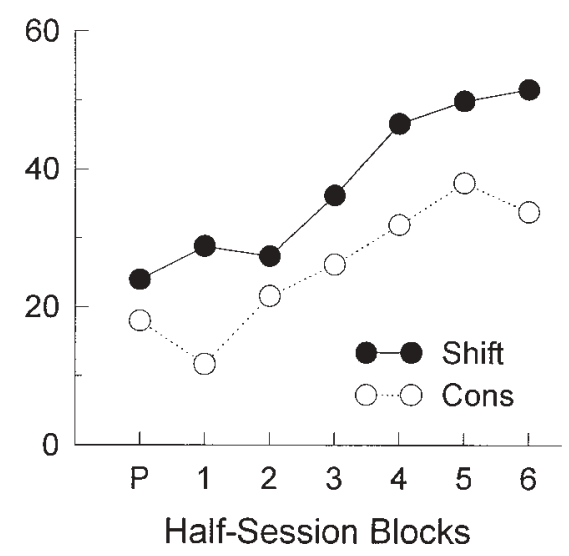

Figure 11. Performance of food cup behavior during a light conditioned stimulus in the test phase of an unpublished experiment conducted by David Bucci. Prior to this test, some rats received a shift in the predictive relation between that light and a tone, whereas for the other rats, a consistent (Cons) relation was maintained. The left panel shows responding of rats that received injections of an immunotoxin into the posterior parietal cortex (PPC), designed to selectively destroy cholinergic projections to that region from the nucleus basalis/substantia innominata, and the right panel shows responding of unlesioned control rats. $P$ on the abscissae refers to responding during the light on the last session of the previous phase. The response was recorded automatically by photocell circuits.

shift in the light-tone contingency, when the discrepancy between the expected and actual outcome was greatest. A difference between shifted and control rats' c-fos activity was observed in the posterior parietal cortex (PPC). Subsequently, Bucci and Michael Conley conducted a series of anatomical tracer studies (unpublished), which verified the connections between the PPC and the large cholinergic neurons in SI, which were presumably destroyed in our SI lesions.

Bucci then conducted a fairly severe test of the hypothesis that the latter connections were critical for the enhanced associability found in the shift condition with the Wilson et al. (1992) task. The immunotoxin ${ }^{192}$ IgG-saporin was injected bilaterally into the PPC. Because that immunotoxin is taken up by receptors on the axon surface as well as those on the cell body, axons (in the PPC) of the SI cholinergic neurons that connect to the PPC should take up the toxin, killing those neurons. But because the toxin was transported into the SI neurons from the PPC, only the SI cholinergic neurons that project to the PPC should be destroyed. In addition, cholinergic neurons whose cell bodies are in the PPC itself apparently do not take up this immunotoxin, so those neurons are not likely to be destroyed. Thus, Bucci's lesion might be expected to selectively destroy cholinergic cells in the SI that also project to the PPC.

In this unpublished experiment, lesioned rats responded like rats with $\mathrm{CN}$ or SI lesions (Figure 11): Rats in the shift condition showed reliably more responding in the test phase than did rats in the consistent training condition. Thus, connections between the SI and PPC are also critical to the enhancement of associability when expectancies are violated.
Summary of incremental processing system. The data described in section 4 show that interruptions of neural processing in a circuit that includes projections from the amygdala $\mathrm{CN}$ to a cholinergic corticopetal system in the basal forebrain interfered with performance in the unblocking and Wilson et al. (1992) serial conditioning tasks. Both tasks are thought to reflect increases in the associability of CSs produced when previously predictable events are made unpredictable. At the same time, behavioral changes often attributed to reductions in CS associability were unaffected by damage to this circuit. Furthermore, damage to this circuitry did not affect either simple conditioning of US-dependent CRs when CS associability changes were not induced (section 2) or aspects of conditioning loosely described as emotional (section 3). Finally, although this incremental processing system overlaps with a system involved in the conditioned potentiation of ORs (section 2) at the level of the amygdala $\mathrm{CN}$, its extension to basal forebrain and cortical regions is not involved in those functions. Thus, it seems reasonable to demand that behavioral theories of conditioning include a mechanism for increases in CS associability that follows rules like those proposed by Pearce and Hall (1980) and operates independently from other changes occurring in conditioning.

\section{B. Septal-Hippocampal System for Decremental, but Not Incremental, Changes in Associability}

Several of the preceding studies suggested that reductions in CS associability were unaffected by damage to structures in the circuit involved in enhancements of CS associability. Many theorists (e.g., Schmajuk \& DiCarlo, 1991), and several investigators, have noted that nonspe- 


\section{Hippocampal Groups}

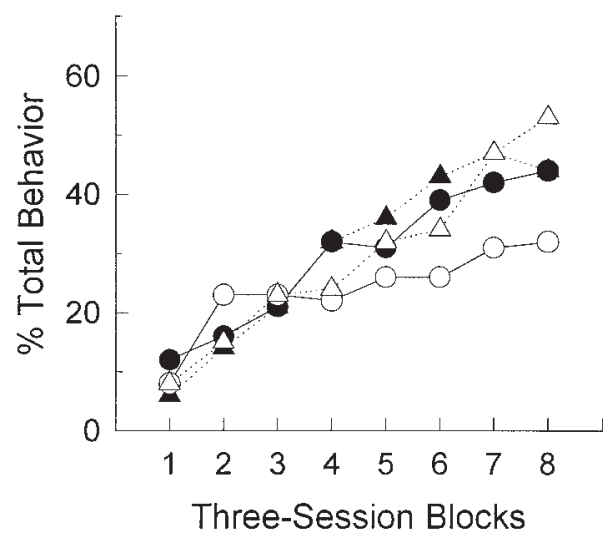

MSANDB Groups

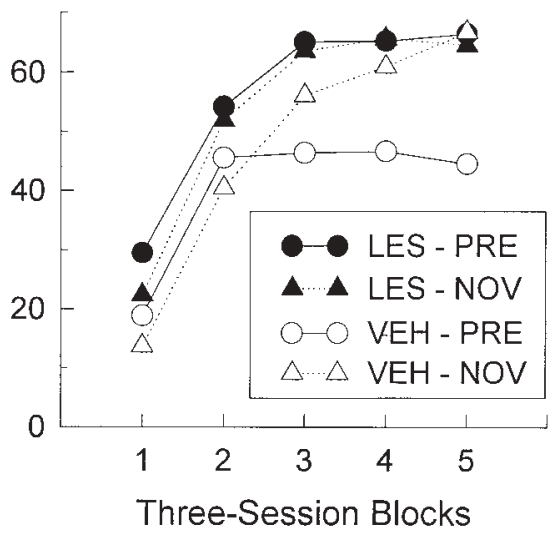

Figure 12. Performance of food cup behavior during two visual conditioned stimuli in the conditioning phase of latent inhibition experiments reported by (left panel) Han, Gallagher, and Holland (1995, Experiment 1) and (right panel) Baxter, Gallagher, and Holland (1997, Experiment 1). The lines labeled LES refer to the performance of rats that had received lesions of either the hippocampus proper (left panel) or the medial septal area and the vertical limb of the diagonal band (MSA/VDB, right panel), and the lines labeled VEH refer to the performance of control rats that received injections of a nontoxic vehicle. NOV refers to the novel visual cue, and PRE refers to the preexposed visual cue.

cific hippocampal lesions disrupt latent inhibition (e.g., Solomon \& Moore, 1975), blocking (e.g., Rickert, Bennet, Lane, \& French, 1978; Solomon, 1977) and other examples of reductions in CS associability during conditioning (Kaye \& Pearce, 1987), although the data are mixed (e.g., Garrud et al., 1984; Honey \& Good, 1993). In this section, I will describe experiments in our labs in which we examined the effects of damage to the hippocampal system on decremental and incremental changes in the associability of CSs. Taken with the parallel experiments described in the preceding section, these studies provide several examples of double dissociations. Reductions in CS associability were affected by hippocampal system damage but not by $\mathrm{CN}-\mathrm{nbm} / \mathrm{SI}$ damage, whereas enhancements in $\mathrm{CS}$ associability were disrupted by $\mathrm{CN}-$ $\mathrm{nbm} / \mathrm{SI}$ damage but not by hippocampal system damage. In addition, they may shed some light on why previous studies of the effects of hippocampal system damage on decreases in associability produced conflicting results.

We examined the behavioral effects of two manipulations of hippocampal system function. In some experiments, we examined the effects of neurotoxic (ibotenic acid) lesions of the hippocampus proper, and in others, the effects of immunotoxic lesions of cholinergic cells in the medial septal area (MSA) and the vertical limb of the diagonal band (VDB). These MSA/VDB lesions effectively cut off the main source of cholinergic input to the hippocampus but leave intact other hippocampal inputs, as well as noncholinergic input from MSA/VDB. The effects of the latter lesions are especially interesting, because Baxter and Gallagher (1996) have recently reported that those lesions do not affect performance in water and radial arm maze tasks - performances that are drastically impaired by lesions of the hippocampus proper.

Effects of hippocampal and MSA/VDB lesions on decrements in CS associability. The existing literature on the effects of hippocampal damage on latent inhibition is mixed. Although most studies done with large, nonselective lesions have yielded reductions in latent inhibition, Honey and Good (1993) found no effects of neurotoxic lesions of hippocampus on latent inhibition: CS preexposure slowed subsequent conditioning in both lesioned and control rats. In our first experiments, we examined the effects of hippocampal (Han, Gallagher, \& Holland, 1995) or MSA/VDB (Baxter, Gallagher, \& Holland, 1997) lesions on latent inhibition with the same experimental procedures that we used in the $\mathrm{CN}$ and $\mathrm{nbm} / \mathrm{SI}$ lesion experiments described earlier. If these lesions interfere with the normal reductions in associability produced when cues are presented without consequence, lesioned rats should show rapid acquisition to both preexposed and novel cues. Figure 12 shows the results of these experiments: both lesions produced precisely that pattern of data. At the same time, neither lesion produced decrements in unconditioned orienting or the habituation of those orienting responses. Indeed the hippocampal lesions seemed to enhance the unconditioned OR to the visual cues, although the slopes of the habituation functions for hippocampally lesioned and control rats did not differ.

It is not clear why our results with hippocampal lesions contradicted those of Honey and Good (1993). The most obvious differences in our respective studies were our use of visual instead of auditory stimuli and our use of withinsubjects rather than between-subjects designs. It might be 
argued that our lesions did not impair latent inhibition but instead discrimination between the two cues. For example, Solomon and Moore (1975) reported that large, nonspecific lesions of the hippocampus broadened the generalization gradient in eyelid conditioning in rabbits.

Unpublished data collected recently in our lab with MSA/VDB-lesioned rats suggest that neither of these factors contributed. First, Mark Baxter found that withinsubjects latent inhibition procedures with auditory cues generated the same outcomes as those procedures did with visual cues. Second, Baxter also found that the lesions did not affect the rate of learning of an explicit discrimination between the two cues, conducted after the conditioning test phase. Third, unlike Solomon and Moore (1975), Javier Morell (1997) found no effect of neurotoxic lesions on the slope of the generalization gradients obtained after training on a single cue.

Effects of hippocampal system damage on performance in blocking. The results of several studies have implicated the hippocampus in blocking (Rickert et al., 1978; Solomon, 1977). In a better controlled study, however, Garrud et al. (1984) failed to find such an effect. Recently, Mark Baxter examined the effects of MSA/VDB lesions on blocking in our Pavlovian conditioning preparation. Unlike Holland and Gallagher's (1993b) study of the effects of $\mathrm{CN}$ lesions on blocking, Baxter used a within-subjects design. After training an A+B - discrimination between two auditory cues (a tone and a noise, counterbalanced), Baxter trained all rats with two compound cues, $\mathrm{AX}+, \mathrm{BY}+$. One compound $(\mathrm{AX})$ comprised the formerly reinforced cue and one novel cue (clicker or light, counterbalanced), and the other compound (BY) comprised the formerly nonreinforced cue and the other novel cue. A final test examined responding to $\mathrm{X}$ and $\mathrm{Y}$, presented nonreinforced. The observation of less responding to $\mathrm{X}$ than to $\mathrm{Y}$ would demonstrate blocking.

The results of the final test depended on the identity of the added cue. The top panel of Figure 13 shows responding to the clicker in the final test. Substantial blocking was observed in both intact and lesioned rats: responding to the clicker was lower when it served as X than when it served as $\mathrm{Y}$ in both cases. Although this difference was numerically smaller among lesioned rats than among control rats, that interaction was not reliable. Thus, the results of this test suggest little (if any) effect of the MSA/VDB lesion on blocking.

However, examination of responding to the light stimulus leads to a different conclusion. The middle panel of Figure 13 shows responding to the light in the final test. Little evidence of conditioning to that light was evident after any of the experimental treatments, as if conditioning to that cue was overshadowed by conditioning to the auditory cues, whether they were previously trained or not. We then conducted a further test of the effects of the blocking procedure on the light cue. At the conclusion of the nonreinforced test sessions, we administered a savings test, in which the rats received light-food pairings for two sessions.

The bottom panel of Figure 13 shows the results of this savings test. Among intact control rats, acquisition of CRs to the light was significantly slower when the light had previously served as X than when it had served as Y. Thus, the savings test revealed a large blocking effect, as was shown with the clicker cue in the nonreinforced test sessions. In contrast, the lesioned rats showed equally rapid acquisition of responding to the light, regardless of its prior treatment. Thus, the results of the savings test, unlike those of the nonreinforced test sessions, showed that the lesions substantially interfered with blocking.

Two accounts for this disparity come to mind. First, it is possible that the MSA/VDB lesions only interfere with blocking with visual cues or with weak cues. A more interesting possibility is that the effects of these lesions on the observation of blocking depend on the extent to which

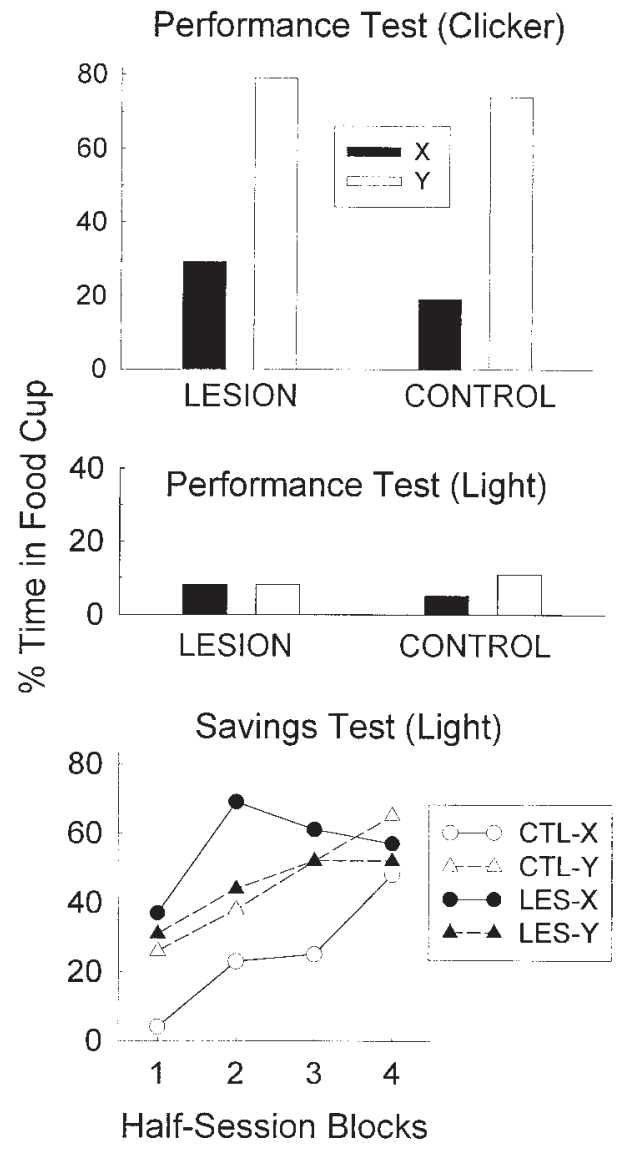

Figure 13. Performance of food cup behavior during the test phases of an unpublished blocking study conducted by Mark Baxter. Rats received training designed to block conditioning to one stimulus $(\mathrm{X})$, but to establish conditioning to another stimulus (Y). For half of the rats, $X$ was a clicker, and $Y$ was a light; and for the other half of the rats, $X$ was the light, and $Y$ was the clicker. The top panel shows responding during nonreinforced presentations of the clicker alone, and the middle panel shows responding during nonreinforced presentations of the light alone. The bottom panel shows responding during the light when it was subsequently paired with food delivery in a savings test. Lesioned (LES) rats had received immunotoxic lesions of the medial septal area and the vertical limb of the diagonal band. CTL refers to unlesioned control rats. The response was recorded automatically by photocell circuits. 
Hippocampal Groups

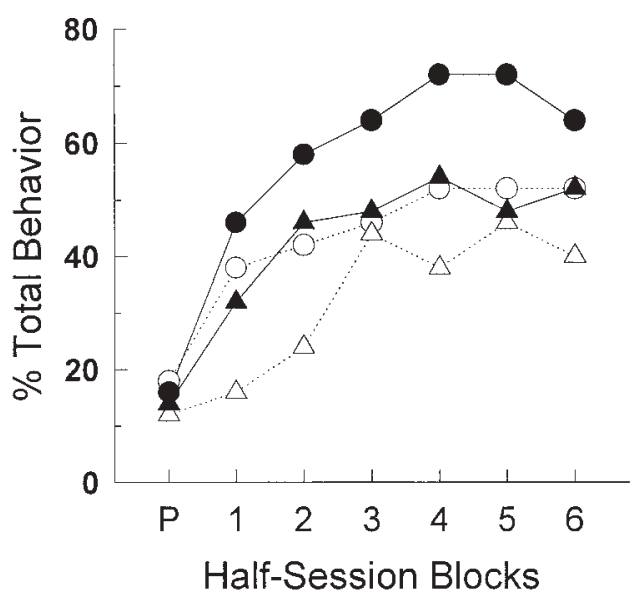

MSANDB Groups

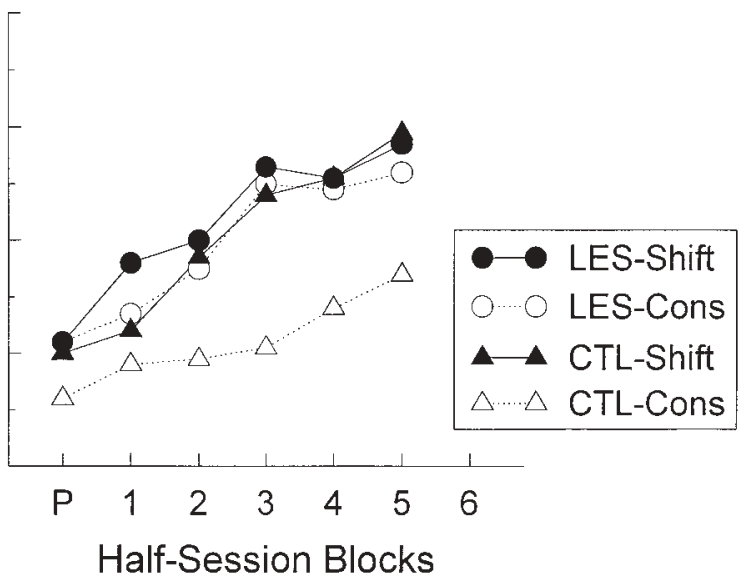

\begin{abstract}
Figure 14. Performance of food cup behavior during a light conditioned stimulus in the test phase of experiments reported by (left panel) Han, Gallagher, and Holland (1995, Experiment 2) and (right panel) Baxter, Gallagher, and Holland (1997, Experiment 2). Prior to this test, some rats received a shift in the predictive relation between that light and a tone, whereas for the other rats, a consistent (Cons) relation was maintained. The lines labeled LES refer to the performance of rats that had received lesions of either the hippocampus proper (left panel) or the medial septal area and the vertical limb of the diagonal band (MSA/VDB, right panel), and the lines labeled CTL refer to the performance of unlesioned control rats. P on the abscissae refers to responding during the light on the last session of the previous phase.
\end{abstract}

the measure of blocking reflects reductions in CS associability, the psychological function that we propose is impaired by these lesions. Recall that the Pearce-Hall (1980) account for blocking, that the associability of the added cue $(\mathrm{X})$ is quickly reduced because the US is already predicted by the blocking cue (A), is only one of many. The Rescorla-Wagner (1972) model, for example, accounts for blocking without postulating any changes in CS associability. Within that model, blocking occurs because the prior training of A renders the US ineffective as a reinforcer when $\mathrm{X}$ is introduced.

Evidence from my laboratory (Holland, 1988) shows that blocking may often reflect both reductions in the effectiveness of the US and reductions in the associability of the added cue. If Rescorla-Wagner (1972) mechanisms rendered the US ineffective in our experiment, the associability of the added $\mathrm{X}$ cue would be largely irrelevant to the observation of conditioned responding in an extinction test: With no effective US, no learning would occur regardless of whether X's associability was driven down or remained high. In contrast, the associability of X would be readily evident when $\mathrm{X}$ was paired with the US in a savings test. Thus, we interpret the results of Baxter's blocking study as suggesting that MSA/VDB lesions interfere with the decrements in CS associability usually produced when trial outcomes are well predicted but do not interfere with variations in US effectiveness. This interpretation also suggests possible explanations for the discrepancies previously observed in the effects of hippocampal damage on blocking. The less that blocking is the consequence of Rescorla-Wagner type processes, the greater the effect that those lesions will have on the observation of blocking in nonreinforced test sessions.

Several interesting predictions are suggested by this interpretation. First, as in Baxter's study, even in experimental settings in which nonreinforced tests of blocking are unaffected by lesions, savings tests might be. Second, performance in the task described by Mackintosh and Turner (1971) and Holland (1985), which was unaffected by $\mathrm{CN}$ lesions (see above), should be altered by hippocampal system lesions. Lesions would prevent the loss in associability of the added cue when that cue was introduced before the shift in the US. Thus, rats with hippocampal damage should not be affected by that intervening compound exposure, essentially as predicted by the Rescorla-Wagner model. Third, rats with MSA/VDB or hippocampal lesions could be used to determine the contribution of processes that produce changes in CSassociability and those that produce variations in USeffectiveness in any given experimental procedure.

Hippocampal lesions and the regulation of associability in serial conditioning. In two experiments, we examined the effects of ibotenate lesions of the hippocampus (Han et al., 1995, Experiment 2) or immunotoxic lesions of the MSA/VDB (Baxter et al., 1997) on performance in the Wilson et al. (1992) serial conditioning task, which we described earlier. According to the Pearce-Hall theory, continued exposure to the consistent light $\rightarrow$ tone relation in the consistent condition results in the gradual reduction of the light's associability. If hippocampal system lesions interfere with those decrements, the light cue should not lose associability for the rats in the consistent condition. 
Consequently, during the light $\rightarrow$ food test phase, the lesioned rats in the consistent condition should outperform intact rats in that condition. Figure 14 shows the results of the test phase of these experiments. In both studies, lesioned rats in the consistent groups acquired food cup responding faster than did the intact consistent rats, and as rapidly as the intact shifted rats. These findings supported the view that the decremental changes in CS processing that occurred with the consistent procedure in intact rats were mediated by hippocampal system circuitry.

Interestingly, hippocampally lesioned rats (but not MSA/VDB-lesioned rats) in the shifted condition showed even more rapid responding than did the lesioned rats in the consistent condition or the intact rats in the shifted condition. Apparently, hippocampal lesions did not interfere with incremental processes engaged by introduction of the inconsistent light-tone relation. Although it might be claimed that the elevated performance of hippocampallesioned rats in both the shifted and consistent conditions suggests a general elevation of learning or conditioned responding, rather than a specific elimination of decremental attentional processes, note that lesioned and intact rats did not differ in their acquisition of conditioning to the tone CS in the first two phases of this study.

A recent unpublished study of unblocking conducted in my laboratory by Greg Fox supports the claim that lesions of the hippocampus proper leave incremental attentional processes intact while simultaneously attenuating decremental processes. In that study, lesioned and control rats first received pairings of a light $\mathrm{CS}$ with a multiple-pellet food US. Then, half the rats received pairings of a light + tone compound with a single-pellet food US, while the other half received the light + tone compound paired with the original multiple-pellet food US. Finally, responding of all rats in the presence of the tone alone was tested in extinction. If hippocampal lesions interfered with the increases in associability thought to be generated by the change in the reinforcer, the lesioned rats in the downshifted group should learn less than the control rats in that group. In fact, the lesioned rats in the downshifted group showed reliably more responding than did the control rats in that group, whereas the lesioned and unlesioned rats in the unshifted control groups showed similar performance. Thus it is unlikely that hippocampal lesions interfered with incremental changes in CS associability. Although interpretation of these results is clouded by the fact that, unlike the lesioned rats, the intact rats failed to show a reliable unblocking effect, the results potentially extend our claim of the independence of decremental and incremental attentional processes. If decremental and incremental processes act independently, the associability of a cue reflects operation of both processes. Elimination of the decremental process would allow the incremental processes to act from a higher baseline of associability and thus enhance unblocking. Implications of this interpretation of Fox's data for construction of a theory of changes in CS associability will be discussed in the final section of this article.
Summary of decremental processing system. The results of the experiments in this section are consistent with our claim that the decremental attentional changes mediated by hippocampal pathways are distinct from the incremental changes mediated by amygdala $\mathrm{CN}$ circuitry. Several phenomena thought to involve reductions in the associability of CSs were affected by destruction of either the hippocampus proper or its cholinergic input from the medial septal area, but not by lesions of either the $\mathrm{CN}$ or the $\mathrm{nbm} / \mathrm{SI}$. Unlike control rats or rats with $\mathrm{CN}$ or $\mathrm{nbm} / \mathrm{SI}$ lesions, rats with hippocampal system damage failed to show retarded acquisition of conditioning to a CS after consistent nonreinforced CS preexposure (latent inhibition), consistent reinforced CS presentations (blocking procedures), or consistent CS-CS compound presentations (Wilson et al., 1992, task). At the same time, hippocampal system damage, unlike $\mathrm{CN}$ or nbm/SI damage, did not disrupt performance in tasks thought to reflect increases in CS associability. Taken together, they suggest a common basis for some of the behavioral deficits observed in latent inhibition, blocking, and serial conditioning tasks, consistent with the general mechanism of reductions in associability proposed by Pearce and Hall (1980).

\section{Implications for Theories of Learning}

Pavlovian conditioning relations produce many changes in the way that animals treat CSs, including the production of various types of CRs, the acquisition of reinforcing power, and the direction of attention toward or away from those stimuli. The data reviewed in this article show that many of these changes involve distinct, relatively independent neural circuitry. First, conditioned orienting behavior is mediated by an amygdalo-nigrostriatal pathway. Second, the enhancement of CS associability produced when expectancies about upcoming events are violated demands the integrity of a circuit that includes projections from the amygdala central nucleus to large cholinergic cells in the nucleus basalis/substantia innominata, which in turn project to cortical areas. Third, the reduction of CS associability produced when stimuli are consistent predictors of other events or are presented without consequence seems dependent on septal-hippocampal circuitry. Fourth, the abilities of CSs to serve as reinforcers for secondorder conditioning and to be sensitive to postconditioning changes in the value of the US are dependent on the basolateral amygdala.

At the same time, none of these circuits is critical for normal acquisition of the most common indicator of Pavlovian conditioning, US-dependent CRs, and each of these functions seems independent of at least some of the others. For example, neither lesions of the basolateral amygdala nor those of the $\mathrm{nbm} / \mathrm{SI}$ disrupt conditioned orienting, and lesions of the amygdala $\mathrm{CN}$ do not compromise second-order conditioning or reinforcer devaluation effects. Similarly, CN lesions do not interfere with the losses in associability observed in, for example, latent inhibition, and hippocampal lesions do not seem to interfere with either conditioned orienting or enhancements in associabil- 
ity induced when the usual event contingencies are altered. Likewise, Javier Morell (1997), working in my laboratory, found no evidence that hippocampal lesions affect secondorder conditioning or reinforcer devaluation effects.

Although the existence of distinct neural circuitry does not necessarily imply independence of behavioral function as well, such dissociation would not be surprising. For example, the independence of neural circuitry for conditioned ORs and US-dependent CRs is consistent with our observations that a variety of procedural manipulations may affect these two classes of behavior differentially (e.g., Holland, 1979a, 1979b, 1980a, 1980b). Such dissociations among various behavioral consequences of conditioning contingencies could have important ramifications for the measurement of associative learning: One's conclusions might depend on one's choice of measure.

In this regard, it is worth describing a recent series of studies by Parkinson, Robbins, and Everitt (1996), who examined the effects of various brain lesions on both sign tracking (approach to and contact with a visual CS source) and goal tracking (approach to the US source) in the appetitive conditioning of rats. They found that rats with amygdala $\mathrm{CN}$ lesions failed to exhibit sign tracking but showed unimpaired goal tracking CRs. At the same time, rats with basolateral amygdala lesions displayed normal levels of both classes of CRs. Thus, their findings with sign- and goal-tracking CRs were analogous to our findings with CS-dependent orienting and US-dependent food cup CRs. It would, then, not be surprising to find signtracking ("autoshaping") and goal-tracking responses to be differentially sensitive to various conditioning parameters, as Pearce and his colleagues (e.g., Pearce \& Collins, 1985; Pearce, Wilson, \& Kaye, 1988) have pointed out. Similarly, to the extent that the reinforcing and response elicitation functions of Pavlovian CSs are subserved by different neural pathways, it would not be surprising if these two consequences of conditioning operations might be more independent than has sometimes been assumed (see, e.g., Rescorla \& Holland, 1976). It is probably naive to think that Pavlovian conditioning contingencies generate a single "association," which somehow feeds into a variety of response production systems. Instead it is likely that many aspects of learning are differentially coded in different systems.

Theories of associability change. The data described here bolster one's confidence that changes in CS associability are involved in producing variations in learning in a variety of conditioning procedures, such as latent inhibition, blocking, unblocking, and various serial conditioning procedures. Most of these studies provide replications of data previously collected in support of a particular theory of changes in CS associability, that of Pearce and Hall (1980). Thus, the data from intact rats reported here generally support their rule for the computation of CS associability: CS associability ( $\alpha_{\mathrm{CS}}$ ) is adjusted to be proportional to the absolute value of the difference between the associative strength supportable by the reinforcer actually presented on a trial and the aggregate prediction of that reinforcer on that trial $\left(\alpha_{\mathrm{CS}} \approx\left|\lambda-V_{\mathrm{Agg}}\right|\right)$.

Nevertheless, the data from rats with various lesions suggest that the mechanisms or rules for increases in CS associability are different from those for decreases. This notion is difficult to reconcile with the simplest statement of the Pearce-Hall (1980) model, in which a single computation $\left(\alpha_{\mathrm{CS}} \approx|\varepsilon|\right.$, where $\left.\varepsilon=\lambda-V_{\mathrm{Agg}}\right)$ directly determines $\alpha_{\mathrm{CS}}$ on the next trial, without specifying the dynamics of how $\alpha_{\mathrm{CS}}$ changes from one value to another. Our data suggest that this error computation must feed into incremental and decremental changes somewhat independently: The impact on $\alpha_{\mathrm{CS}}$ of the same error $(\varepsilon)$ differed drastically, depending on the nature of the lesion. Consider, for example, rats with amygdala $\mathrm{CN}$ lesions. These rats were unable to adjust $\alpha_{\mathrm{CS}}$ upward when $|\varepsilon|$ increased, but they were unaffected in their ability to adjust $\alpha_{\mathrm{CS}}$ downward when $|\varepsilon|$ was small. In contrast, rats with hippocampal lesions could adjust $\alpha_{\mathrm{CS}}$ upward when $|\varepsilon|$ increased, but were unable to adjust $\alpha_{\mathrm{CS}}$ downward when $|\varepsilon|$ was small. In each case, the rats could use the error to adjust $\alpha_{\mathrm{CS}}$, but only in one direction. So it seems reasonable to require that a behavioral theory of changes in associability separately describes the rules for increases and decreases in CS associability.

At the same time, it would also be desirable for such a theory to provide a more satisfactory account of the dynamics of those changes than was initially provided in the Pearce-Hall (1980) model. In that model, a change in the reinforcer produced an instantaneous and complete increase in $\alpha_{\mathrm{CS}}$, but consistent delivery of the same reinforcer produced gradually declining $\alpha_{\mathrm{CS}}$ values (as the predicted US value approached the actual US value). Although considerable data from both Pearce's and my labs suggest that adjustments in $\alpha_{\mathrm{CS}}$ after reinforcer changes are more rapid than those after associated with consistent delivery of the same events, they are clearly not instantaneous (e.g., Kaye $\&$ Pearce, 1984). For example, I have found that the magnitude of the shift advantage in the Wilson et al. (1992) task is increased with the amount of shift training up to about five sessions. One way of dealing with this evidence of gradual associability changes, first suggested by Pearce and Hall (1980), is simply to demand that the calculation of $\alpha_{\mathrm{CS}}$ be based on predictions of the US averaged over several previous trials, yielding more gradual changes.

However, many approaches to framing rules for associability changes deal with both issues of dynamics and the possible independence of incremental and decremental changes. Perhaps the simplest is to assume that the change in associability $(\Delta \alpha)$, rather than $\alpha$ itself, is proportional to the error, $\Delta \alpha_{\mathrm{CS}}=c|\varepsilon|$ (where $c$ is some proportionality constant). This approach generates more gradual, negatively accelerated increases in $\alpha$ when the reinforcer is altered, rather than instantaneous ones. Of course, unlike Pearce and Hall's rule, this rule provides no opportunity for decreases in associability; indeed, once $\alpha$ was driven up by surprise, it would never return to lower 
values, even if the error returned to zero. Thus, this approach demands that a separate mechanism concomitantly acts to reduce $\alpha$, an assumption that is consistent with the data described in this article.

The simplest rule for decreases in $\alpha$ might be one in which $\alpha$ decreases with each presentation of the CS, regardless of its consequence; for example, $\Delta \alpha=d(a-\alpha)$. In this expression, $a$, some minimum value for $\alpha$, is introduced to avoid the embarrassment of a negative associability value (and $d$ is a proportionality constant for decreases in associability). Thus, considering both incremental and decremental functions, $\Delta \alpha=c|\varepsilon|+d(a-\alpha)$, or, substituting, $\Delta \alpha=c|\varepsilon|-d(\alpha-a)$. Alternately, the decreases in $\alpha$ might also be (inversely) proportional to error, so that $\Delta \alpha=c|\varepsilon|-[d(\alpha-a) /|\varepsilon|]$ (with an obvious problem with zero error). Within either of these approaches, $\alpha$ would decrease continuously with repeated exposure to the same event contingencies, and changes in those contingencies (increases in $|\varepsilon|$ ) would increase $\alpha$ from its current value.

The choice of a decremental rule like the latter one is favored by our failures to find effects of either $\mathrm{CN}$ lesions or septal-hippocampal lesions on the acquisition of USdependent CRs in simple conditioning procedures. If simple presentation of an event is sufficient to reduce its associability, the associability of a CS should decrease during consistent CS-US pairings, thus slowing the rate of conditioning in intact rats. In the absence of such a decremental process, lesioned rats should then acquire CRs more rapidly than should intact rats, especially as conditioning proceeds. We have never observed such an effect with simple conditioning procedures, though the results of our latent inhibition, blocking, and serial conditioning experiments (described in this article) all suggest that consistent training contingencies engage a hippocampally dependent decremental process. However, if decreases in $\alpha$ were negligible until $\varepsilon$ was close to zero, simple conditioning would already be well established before any effect of hippocampal lesions on $\alpha$ could occur. In contrast, in blocking procedures, the added cue is introduced only after $\varepsilon$ has been driven close to zero, so decremental changes in $\alpha$ at the point would be substantial. Similarly, in the Wilson et al. (1992) procedure, training is continued long after conditioning (and presumably light-tone learning) has reached asymptote. Indeed, unpublished evidence from our lab shows that the rate of acquisition in the test phase after consistent training in this task is inversely related to the amount of consistent training. And, in the preexposure phase of the latent inhibition task, the discrepancy between expected and obtained outcomes is zero from the very beginning.

Thus, it seems reasonable to speculate that a basic error correction process is critical to several dynamic aspects of conditioning, including both incremental and decremental changes in associability and changes in the effectiveness of the US. Because of the highly selective effects of each lesion described here, none of them can have its effect by interfering with a single calculation of $\varepsilon$. It remains to be seen whether each of these systems has access to a single error correction routine, or each system separately calculates $\varepsilon$.

A further constraint on a theory of changes in the associability of CSs is suggested by our failure to find an effect of $\mathrm{CN}$ lesions on simple CR acquisition. Initial CS-US pairings should produce a large value for $\varepsilon$, thereby engaging the $\mathrm{CN}$-dependent incremental system. Consequently, intact rats should maintain higher $\alpha$ values earlier in conditioning than rats with $\mathrm{CN}$ lesions, and thus more rapid conditioning, which we have never observed. One solution to this problem is to adopt Pearce and Hall's (1980) view that $\alpha$ never increases beyond its initial, intrinsic value. For example, one could modify the rule for $\mathrm{CS}$ increases so that they would be proportional to the difference between the current and initial $\left(\alpha_{\mathrm{i}}\right)$ values of $\alpha$, $\Delta \alpha=c\left(\alpha_{\mathrm{i}}-\alpha\right)|\varepsilon|$.With this additional constraint, the impact of the unavailability of an incremental mechanism in $\mathrm{CN}$-lesioned rats on the rate of simple conditioning would be minimal.

However, other evidence suggests that the addition of this constraint is not sufficient. For example, reduction in $\alpha$ before simple conditioning should make an incremental mechanism important again. Thus, in a latent inhibition experiment, the preexposure-decremented $\alpha$ should be rapidly readjusted during the conditioning phase in intact rats, but not in CN-lesioned rats. So, compared with intact rats, $\mathrm{CN}$-lesioned rats should show enhanced latent inhibition, because they would be unable to readjust $\alpha$ upward. Although it could be argued that the latent inhibition effects observed in our laboratories are small and perhaps insensitive to further increases, it must be noted that we have no evidence that either $\mathrm{CN}$ or $\mathrm{nbm} / \mathrm{SI}$ lesions enhance latent inhibition effects.

Another approach to dealing with the lack of a $\mathrm{CN}$ lesion effect on simple conditioning, either with or without $\mathrm{CS}$ preexposure, is to assume that the $\mathrm{CN}$-dependent incremental process is engaged only when $\varepsilon$ is negativethat is, only when the outcome of a trial is overexpected. Within this approach, the CN-dependent incremental process would not be engaged in simple conditioning, whatever the initial value of $\alpha$, so $\mathrm{CN}$ lesions would have no impact on US-dependent CRs. Likewise, $\mathrm{CN}$ lesions would not be anticipated to affect the results of changes inevent contingencies that produce underexpectations of the trial outcome. Recall that Holland and Gallagher (1993a) found that although CN lesions eliminated unblocking when the value of the US was shifted downward (overexpectation), they did not affect unblocking when the value was shifted upward (underexpectation). Because unblocking with upshifts in the US could be attributed to the enhanced reinforcement value of the upshifted US (Rescorla \& Wagner, 1972), it is intriguing to speculate that increases in CS associability might occur only when trial outcomes are overexpected. We have no further evidence concerning this interesting issue, but it is worth noting that other procedures that seem to induce increases in CS associability (e.g., Hall \& Pearce, 1982; Swan \& 
Pearce, 1988) also involve downshifts in US value. ${ }^{1}$ At the same time, note that elsewhere (Holland, 1988) I have presented evidence that upshifts in US value in unblocking experiments produce changes in both US strength and CS associability. It would be valuable to examine the effects of CN lesions across procedures that induce different proportions of each of these kinds of changes. Of course, it is also possible that there are multiple mechanisms for enhancing $\alpha$, and that only the $\mathrm{CN}$-dependent mechanism is limited to overexpectation effects.

Relation of associability changes to selective attention. The attentional processes that I have emphasized in this article are limited to changes in the ability of CSs to participate in new learning. In contrast, a more typical concern in the study of attention is the issue of stimulus selection. An enormous amount of information is available to animals, but at any moment only part of it can be effectively processed. For most researchers, the mechanisms of selecting some items in an array for further processing and excluding other items defines the problem of attention. Within some attentional theories of conditioning (see, e.g., Sutherland \& Mackintosh, 1971), changes in associability are often closely related to selection. For example, in Sutherland and Mackintosh's (1971) theory, variables that affect attention do so by making the use of particular stimulus "analyzers" more likely. Thus, a cue that demands more processing at the time of learning, and so is more associable, will also demand more processing at the time of performance, at the expense of processing other cues.

In contrast, Pearce and Hall's (1980) theory, although not explicitly addressing the issue, must posit an indirect relation between associability changes and selection. Highly predictive CSs must surely continue to control behavior, despite severe reductions in their associability. Indeed, Pearce and Hall distinguished between "controlled" attentional processing, relating to associability, and "automatic" attentional processing of CSs, which is important at the time of performance. Unfortunately, Pearce and Hall did not propose rules for automatic processing. Thus, in terms of specifying performance rules, Pearce and Hall's model is no different from that of Rescorla and Wagner (1972), which has no provision for variations in CS processing of any kind. Nevertheless, many problems of stimulus selection can be handled simply by noting that behavior would be controlled by various CSs proportional to their associative strengths. For example, highly salient cues would acquire conditioning very rapidly and so would quickly come to overshadow less salient cues that accompanied them. Indeed, part of the success of the Rescorla-Wagner model was its ability to provide a nonattentional framework for the interpretation of many conditioning phenomena initially described as involving selective attention.

For the most part, continued development of theories of processing of arrays of CSs in performance has concerned perceptual analyses of part-whole or element- configuration relations (e.g., Cook, Riley, \& Brown, 1992; Lamb, 1988; Pearce, 1994) and has made little contact with the issues of changes in associability important to models like Pearce and Hall's (1980). At the same time, investigations of the neurobiology of attention have generated some data that suggest that brain systems that we have implicated in enhancements of CS associability are also involved in performance in well-learned choice tasks. For example, Chiba, Bushnell, Oshiro, and Gallagher (1995) found that lesions of nbm/SI disrupted performance in a spatial orienting task in which rats had to respond to one of two spatially separated cues after a prior cuing at one of the target visual locations. Similarly, Muir et al. (1992) found that nbm lesions disrupted rats' performance in a five-choice serial reaction time task, in which rats were forced to approach a series of light sources illuminated in random order. Unfortunately, the relation of any of these tasks to changes in associability is unknown.

Lesioned rats as tools in developing theories of learning. The most popular theories of conditioning have attempted to account for variations in behavior across a variety of conditioning procedures in terms of variations in a small number of underlying processes. For example, the Rescorla-Wagner (1972) model permitted variations in the effectiveness of the reinforcer, but not in the associability of the CS. Likewise, the Pearce-Hall (1980) model permitted CS associability to vary, but not the effectiveness of the US. Limiting the effects of procedural manipulations to a single underlying process permitted the straightforward development of simple quantitative models. To the extent that conditioning procedures differentially engage several interacting processes, that task is made more difficult. The theorist must ask (for example), what is the weighting of manipulation X's effects on increases and decreases in CS associability and on the effective value of the reinforcer? Lesion methods like those described in this paper may provide a useful tool in this endeavor, by providing "reduced preparations" (Teitelbaum \& Pellis, 1992) - that is, rats that lack one or more of these putative contributory processes. Thus, as noted earlier, rats with $\mathrm{CN}$ or $\mathrm{nbm} / \mathrm{SI}$ lesions, which fail to show increased CS associability when expectancies about upcoming events are violated, may be useful subjects for investigations of the effects of such violations on the effectiveness of USs. Likewise, rats with medial septal or hippocampal lesions, which fail to show reductions in CS associability, might contribute in analogous ways. It might be argued that rats with lesions of both the CN-nbm/SI and septal-hippocampal systems would be ideal subjects for the examination of the effects of various manipulations on US effectiveness, as specified by models like that of Rescorla and Wagner (1972). Baxter, Bucci, Holland, and Gallagher (1996) recently examined the performance of rats with lesions of both systems in several tasks described in this article. Unfortunately, their results were difficult to interpret, because in many cases these lesions, unlike the others described here, produced general deficits in condi- 
tioning. As noted earlier, the more pervasive the deficits produced by a lesion, the less precisely that lesion can be used as an analytic tool in investigations like these.

Conclusion. Pavlovian conditioning relations produce a number of changes in CS processing. These changes include (but are not limited to) the generation of a variety of classes of conditioned behavior, the acquisition of new motivational significance (e.g., reinforcement power), and alterations in the ability of those cues to participate in future learning. Most learning theories have not addressed this multiplicity of functions explicitly, tacitly assuming substantial correlation among them. Observations that these functions are served by different brain systems encourage the belief that they may have distinguishable determinants at the behavioral level as well.

The bulk of the data described in this article concern changes in CS associability during Pavlovian conditioning. Although in this paper we have not attempted to describe a new quantitative model for changes in associability, many data described may potentially constrain the nature of such a model. Our major contribution in this regard is the suggestion that increases and decreases in associability should be represented separately in such a model. Second, we have suggested that both incremental and decremental changes in associability involve a calculation of $\varepsilon$, the discrepancy or error between anticipated and actual trial outcomes. Third, we have suggested that overexpectations of upcoming events - that is, the omission or degrading of those events - may affect associability differently than underexpectations. Each of these suggestions has implications for the results of purely behavioral experiments and could have arisen from other, purely behavioral experiments. That is, the lesion strategy is in no way necessary for this endeavor. But it has provided valuable data, and it is likely to serve as a useful tool for subsequent investigations of these issues. Comparisons of intact rats and rats with various lesions might provide better opportunities to derive parameter values for the functions defining associability and reinforcer value and to determine the conditions under which incremental or decremental changes in associability occur.

The continued development of contemporary models of learning is important for understanding the neural basis of psychological constructs such as attention and reinforcement. Brain function is often inferred from the effects of lesions on, or from measures of brain activity during, performance in particular conditioning tasks. But the value of these inferences is limited if the behavioral and psychological bases of those performances are not well understood. At the same time, investigations of brain function can inform learning theory both by providing additional tools for examining component behavioral processes and by providing bases for theoretical process distinctions at another level of analysis.

\section{REFERENCES}

Baxter, M., Bucci, D. J., Holland, P. C., \& Gallagher, M. (1996). Combined selective lesions of both septohippocampal and corticopetal basal forebrain cholinergic projections disrupt attentional pro- cessing but not spatial learning. Society for Neuroscience Abstracts, 22, 681

BaXter, M. G., \& Gallagher, M. (1996). Intact spatial learning in both young and aged rats following selective removal of hippocampal cholinergic input. Behavioral Neuroscience, 110, 460-467.

BaXter, M. G., Gallagher, M., \& Holland, P. C. (1997). Disruption of attentional processing by selective removal of hippocampal cholinergic input. Journal of Neuroscience, 17, 5230-5236.

Bucci, D. J., Chiba, A. A., Holland, P. C., \& Gallagher, M. (1995). c-Fos induction during an incremental change in attention. Society for Neuroscience Abstracts, 21, 935.

CAdor, M., Robbins, T. W., \& Everitt, B. J. (1989). Involvement of the amygdala in stimulus-reward associations: Interaction with the ventral striatum. Neuroscience, 30, 77-86.

Carli, M., Jones, G. H., \& Robbins, T. W. (1989). Effects of unilateral dorsal and ventral striatal dopamine depletion on visual neglect in the rat: A neural and behavioural analysis. Neuroscience, 29, 309-327.

Chiba, A. A., Bucci, D. J., Holland, P. C., \& Gallagher, M. (1995). Basal forebrain cholinergic lesions disrupt increments but not decrements in conditioned stimulus processing. Journal of Neuroscience, 15, 7315-7322

Chiba, A. A., Bushnell, P. J., Oshiro, W. M., Gallagher, M. (1995). Altered selective attention in rats with cholinergic lesions of the substantia innominata. Society for Neuroscience Abstracts, 21, 936.

Collins, L., \& Pearce, J. M. (1985). Predictive accuracy and the effects of partial reinforcement on serial autoshaping. Journal of Experimental Psychology: Animal Behavior Processes, 11, 548-564.

Cook, R. G., Riley, D. A., \& Brown, M. F. (1992). Spatial and configural factors in compound stimulus processing by pigeons. Animal Learning \& Behavior, 20, 41-55.

DAvis, M. (1992). The role of the amygdala in conditioned fear. In J. P. Aggleton (Ed.), The amygdala: Neurological aspects of emotion, memory, and mental dysfunction (pp. 255-306). Chichester, U.K.: Wiley.

Dickinson, A., Hall, G., \& MaCKInTOSH, N. J. (1976). Surprise and the attenuation of blocking. Journal of Experimental Psychology: Animal Behavior Processes, 2, 313-322.

EDELINE, J.-M., \& WeINBERGER, N. M. (1992). Associative retuning in the thalamic source of input to the amygdala and auditory cortex: Receptive field plasticity in the medial division of the medial geniculate body. Behavioral Neuroscience, 196, 81-105.

Gallagher, M., \& Chiba, A. (1996). The amygdala and emotion. Current Opinion in Neurobiology, 6, 221-227.

Gallagher, M., Graham, P. W., \& Holland, P. C. (1990). The amygdala central nucleus and appetitive Pavlovian conditioning: Lesions impair one class of conditioned performance. Journal of Neuroscience, 10, 1906-1911.

Gallagher, M., \& Holland, P. (1992). Understanding the function of the central nucleus: Is simple conditioning enough? In J. P. Aggleton (Ed.), The amygdala: Neurobiological aspects of emotion, memory, and mental dysfunction (pp. 307-321). Chichester, U.K.: Wiley.

Gallagher, M., \& Holland, P. (1994). The amygdala complex: Multiple roles in associative learning and attention. Proceedings of the $\mathrm{Na}$ tional Academy of Sciences, 91, 11771-11776.

Garrud, P., Rawlins, J. N. P., Mackintosh, N. J., Goodal, G., Cotton, M. M., \& FELDON, J. (1984). Successful overshadowing and blocking in hippocampectomized rats. Behavioural Brain Research, 12, 39-53.

Grove, E. A. (1988). Neural associations of the substantia innominata in the rat: Afferent connections. Journal of Comparative Neurology, 277, 315-346

Hall, G., \& Pearce, J. M. (1982). Restoring the associability of a preexposed CS by a surprising event. Quarterly Journal of Experimental Psychology, 34B, 127-140.

Han, J.-S., Gallagher, M., \& Holland, P. C. (1995). Hippocampal lesions disrupt decrements but not increments in conditioned stimulus processing. Journal of Neuroscience, 11, 7323-7329.

Han, J.-S., McMahan, R. W., Holland, P. [C.], \& Gallagher, M. (1997). The role of an amygdalo-nigrostriatal pathway in associative learning. Journal of Neuroscience, 17, 3913-3919.

Hatfield, T. Han, J.-S., Conley, M., Gallagher, M., \& Holland, P. [C.] (1996). Neurotoxic lesions of basolateral, but not central, amygdala interfere with Pavlovian second-order conditioning and reinforcer devaluation effects. Journal of Neuroscience, 16, 5256-5265. 
Heimer, L., \& Alheid, G. F. (1991). Piecing together the puzzle of forebrain anatomy. In T. C. Napier, P. W. Kalivas, \& I. Hanin (Eds.), The basal forebrain (pp. 1-42). New York: Plenum.

Henke, P. G., \& MAXwell, D. (1973). Lesions in the amygdala and the frustration effect. Physiology \& Behavior, 10, 647-650.

Hiтchсоск, J. M., \& Davis, M. (1991). Efferent pathway of the amygdala involved in conditioned fear as measured by the fear-potentiated startle paradigm. Behavioral Neuroscience, 105, 826-842.

Holland, P. C. (1977). Conditioned stimulus as a determinant of the form of the Pavlovian conditioned response. Journal of Experimental Psychology: Animal Behavior Processes, 3, 77-104.

Holland, P. C. (1979a). Differential effects of omission contingencies on various components of Pavlovian appetitive conditioned behavior in rats. Journal of Experimental Psychology: Animal Behavior Processes, 5, 178-193.

Holland, P. C. (1979b). The effects of qualitative and quantitative variation in the US on individual components of Pavlovian appetitive conditioned behavior in rats. Animal Learning \& Behavior, 7, 424-432.

Holland, P. C. (1980a). CS-US interval as a determinant of the form of Pavlovian appetitive conditioned responses. Journal of Experimental Psychology: Animal Behavior Processes, 6, 155-174.

Holland, P. C. (1980b). Influence of visual conditioned stimulus characteristics on the form of Pavlovian appetitive conditioned responding in rats. Journal of Experimental Psychology: Animal Behavior Processes, 6, 81-97.

Holland, P. C. (1984a). The origins of Pavlovian conditioned behavior. In G. H. Bower (Ed.), The psychology of learning and motivation (Vol. 18, pp. 129-173). Englewood Cliffs, NJ: Prentice-Hall.

Holland, P. C. (1984b). Unblocking in Pavlovian appetitive conditioning. Journal of Experimental Psychology: Animal Behavior Processes, 10, 476-497.

Holland, P. C. (1985). Pretraining a compound conditioned stimulus reduces unblocking. Bulletin of the Psychonomic Society, 23, 237-240.

Holland, P. C. (1988). Excitation and inhibition in unblocking. Journal of Experimental Psychology: Animal Behavior Processes, 14, 261-279.

Holland, P. C. (1990). Event representation in Pavlovian conditioning: Image and action. Cognition, 37, 105-131.

Holland, P. C., \& Gallagher, M. (1993a). Amygdala central nucleus lesions disrupt increments, but not decrements, in CS processing. Behavioral Neuroscience, 107, 246-253.

Holland, P. C., \& Gallagher, M. (1993b). Effects of amygdala central nucleus lesions on blocking and unblocking. Behavioral Neuroscience, 107, 235-245.

Holland, P. C., \& Straub, J. J. (1979). Differential effects of two ways of devaluing the unconditioned stimulus after Pavlovian appetitive conditioning. Journal of Experimental Psychology: Animal Behavior Processes, 5, 65-78.

HoneY, R. C., \& Good, M. (1993). Selective hippocampal lesions abolish the contextual specificity of latent inhibition and conditioning. Behavioral Neuroscience, 107, 23-33.

KAMIN, L. J. (1968). Attention-like processes in classical conditioning. In M. R. Jones (Ed.), Miami symposium on the prediction of behavior: Aversive stimulation (pp. 9-32). Coral Gables, FL: University of Miami Press.

Kapp, B. S., Pascoe, J. P., \& Bixler, M. A. (1984). The amygdala: A neuroanatomical systems approach to its contribution to aversive conditioning. In L. Squire \& N. Butters (Eds.), The neuropsychology of memory (pp. 473-488). New York: Guilford.

Kapp, B. S., Supple, W. F., JR., \& Whalen, P. J. (1994). Effects of electrical stimulation of the amygdaloid central nucleus on neocortical arousal in the rabbit. Behavioral Neuroscience, 108, 81-93.

Kaye, H., \& Pearce, J. M. (1984). The strength of the orienting response during Pavlovian conditioning. Journal of Experimental Psychology, 10, 90-109.

KaYe, H., \& Pearce, J. M. (1987). Hippocampal lesions attenuate latent inhibition of a CS and of a neutral stimulus. Psychobiology, 15, 293-299.

Kemble, E. D., \& Beckman, G. J. (1970). Runway performance of rats following amygdaloid lesions. Physiology \& Behavior, 5, 45-47.

LAMB, M. (1988). Selective attention: Effects of cuing on the processing of different types of compound stimuli. Journal of Experimental Psychology: Animal Behavior Processes, 14, 96-104.
LeDoux, J. E. (1992). Emotion and the amygdala. In J. P. Aggleton (Ed.), The amygdala: Neurobiological aspects of emotion, memory, and mental dysfunction (pp. 339-351). Chichester, U.K.: Wiley.

LeDoux, J. E., Iwata, J., Cicchetti, P., \& Reis, D. J. (1988). Different projections of the central amygdaloid nucleus mediate autonomic and behavioral correlates of conditioned fear. Journal of Neuroscience, $\mathbf{8}$, 2517-2529.

Lubow, R. E., Wagner, M., \& Weiner, I. (1982). The effects of compound stimulus preexposure of two elements differing in salience on the acquisition of conditioned suppression. Animal Learning \& Behavior, 10, 483-489.

Luria, A. R. (1966). Higher cortical functions in man (B. Haigh, Trans.). New York: Basic Books.

MackinTosh, N. J. (1975). A theory of attention: Variations in the associability of stimuli with reinforcement. Psychological Review, 82, 276-298.

Mackintosh, N. J., \& Turner, C. (1971). Blocking as a function of novelty of CS and predictability of UCS. Quarterly Journal of Experimental Psychology, 23, 359-366.

McDonald, R. J., \& White, N. M. (1993). A triple dissociation of memory systems: Hippocampus, amygdala, and dorsal striatum. Behavioral Neuroscience, 107, 3-22.

MoReLL, J. R. (1997). The hippocampus: S-R, S-S, and spatial learning. Unpublished doctoral dissertation, Duke University.

Mowrer, O. H. (1960). Learning theory and behavior. New York: Wiley.

Muir, J. L., Dunnett, S. B., Robbins, T. W., \& Everitt, B. J. (1992). Attentional functions of the forebrain cholinergic systems: Effects of intraventricular hemicholinium, physostigmine, basal forebrain lesions and intracortical grafts on a multiple choice serial reaction time task. Experimental Brain Research, 89, 611-622.

Parkinson, J. A., Robbins, T. W., \& Everitt, B. J. (1996). Lesions of the nucleus accumbens core, but not basolateral amygdala or subiculum, disrupt stimulus-reward learning in a novel autoshaping procedure. Society for Neuroscience Abstracts, 22, 1118 .

Pearce, J. M. (1994). Similarity and discrimination: A selective review and a connectionist model. Psychological Review, 101, 587-607.

Pearce, J. M., \& Collins, L. (1985). The effect of informative events on response rates. Quarterly Journal of Experimental Psychology, 34B, 49-60.

Pearce, J. M., \& Hall, G. (1980). A model for Pavlovian learning: Variations in the effectiveness of conditioned but not of unconditioned stimuli. Psychological Review, 106, 532-552.

Pearce, J. M., Wilson, P., \& Kaye, H. (1988). The influence of predictive accuracy on serial conditioning in the rat. Quarterly Journal of Experimental Psychology, 40B, 181-198.

Peterson, S. E., Robinson, D. L., \& Currie, J. N. (1989). Influences of lesions of parietal cortex on visual spatial attention. Neuropsychology, 25, 97-105.

Posner, M. I., Walker, J. A., Friedrich, F. J., \& Rafal, R. D. (1984). Effects of parietal injury on covert orienting of attention. Journal of Neuroscience, 4, 1863-1874.

Price, J. L., \& Amaral, D. G. (1981). An autoradiographic study of the projections of the central nucleus of the monkey amygdala. Journal of Neuroscience, 11, 1242-1259.

Rescorla, R. A. (1980). Second-order conditioning. Hillsdale, NJ: Erlbaum.

Rescorla, R. A. (1991). Separate reinforcement can enhance the effectiveness of modulators. Journal of Experimental Psychology: Animal Behavior Processes, 17, 259-269.

Rescorla, R. A., \& Holland, P. C. (1976). Some behavioral approaches to the study of learning. In E. Bennett \& M. R. Rozensweig (Eds.), Neural mechanisms of learning and memory (pp. 165-192). Cambridge, MA: MIT Press.

Rescorla, R. A., \& Solomon, R. L. (1967). Two-process learning theory: Relationships between Pavlovian conditioning and instrumental learning. Psychological Review, 74, 151-182.

Rescorla, R. A., \& Wagner, A. R. (1972). A theory of Pavlovian conditioning: Variations in the effectiveness of reinforcement and nonreinforcement. In A. H. Black \& W. F. Prokasy (Eds.), Classical conditioning II: Current research and theory (pp. 64-99). New York: Appleton-Century-Crofts.

Rickert, E. J., Bennett, T. L., Lane, P., \& French, J. (1978). Hip- 
pocampectomy and the attenuation of blocking. Behavioral Biology, 22, 147-160.

Robledo, P., Robbins, T. W., \& Everitt, B. J. (1996). Effects of excitotoxic acid lesions of the central amygdaloid nucleus on the potentiation of reward-related stimuli by intra-accumbens amphetamine. Behavioral Neuroscience, 110, 981-990.

SalzmanN, E. (1995). Attention and memory trials during neuronal recording from the primate pulvinar and posterior parietal cortex (area PG). Behavioural Brain Research, 67, 241-253.

SchmajuK, N. A., \& DiCarlo, J. J. (1991). A neural network approach to hippocampal function in classical conditioning. Behavioural Neuroscience, 105, 82-110.

Solomon, P. (1977). Role of the hippocampus in blocking and conditioned inhibition of the rabbit's nictitating membrane response. Journal of Comparative \& Physiological Psychology, 91, 407-417.

Solomon, P., \& Moore, J. W. (1975). Latent inhibition and stimulus generalization of the classically conditioned nictitating membrane response in rabbits (Oryctolagus cuniculus) following dorsal hippocampal ablation. Journal of Comparative \& Physiological Psychology, 89, 1192-1203.

Staddon, J. E. R. (1983). Adaptive behavior and learning. Cambridge: Cambridge University Press.

Sutherland, N. S., \& Mackintosh, N. J. (1971). Mechanisms of animal discrimination and learning. New York: Academic Press.
Swan, J. A., \& Pearce, J. M. (1988). The orienting response as an index of stimulus associability in rats. Journal of Experimental Psychology: Animal Behavior Processes, 14, 292-301.

Teitelbaum, P., \& Pellis, P. M. (1992). Toward a synthetic physiological psychology. Psychological Science, 3, 4-20.

Weingarten, H. P. (1983). Conditioned cues elicit feeding in sated rats: A role for learning in meal initiation. Science, 220, 431-433.

Wilson, P. N., Boumphrey, P., \& Pearce, J. M. (1992). Restoration of the orienting response to a light by a change in its predictive accuracy. Quarterly Journal of Experimental Psychology, 44B, 17-36.

\section{NOTE}

1. Note that the version of the Hall-Pearce negative transfer task that examines the effect of an upshift in the US assesses $\alpha$ by examining the rate of extinction after a transient increase in reinforcer magnitude $(\mathrm{L}+|\mathrm{L}++| \mathrm{L}-$ vs. $\mathrm{L}+|\mathrm{T}++| \mathrm{L}-)$. The increased associability of $\mathrm{L}$ in this example might be produced by the positive error when the reinforcer is introduced in the second phase or the negative error generated on the initial extinction trials.

(Manuscript received March 28, 1997; revision accepted for publication June 20,1997.) 